Rev. 0

Key Words: Dose Conversion Factor, Maximum Contaminant Level, Special Analysis

Retention: Permanent

\title{
Radionuclide Data Package for Performance Assessment Calculations Related to the E-Area Low-Level Waste Facility at the Savannah River Site
}

\author{
James R. Cook
}

March 20, 2007

Savannah River National Laboratory

Washington Savannah River Company

Savannah River Site

Aiken. SC 29808

Prepared for the U.S. Department of Energy Under

Contract Number DE-AC09-96SR18500 


\section{REVIEWS AND APPROVALS}

\section{Author}

James R. Cook, Author, SRNL

Date

\section{Approvals and Reviews}

Elmer L. Wilhite, Internal Review (per WSRC-IM-2002-00011, Rev. 2), SRNL Date

Maurice J.Ades, Internal Design Check (per WSRC-IM-2002-00011, Rev. 2,) WMAP Date

B.T. Butcher, Level 4 Manager, SRNL

Date

W. E. Stevens, Level 3 Manager, SRNL

Date

W. T. Goldston, Waste Management Area Project

Date 
Key Words: Key Words: Dose Conversion Factor, Maximum Contaminant Level, Special Analysis

Retention: Permanent

\title{
Radionuclide Data Package for Performance Assessment Calculations Related to the E-Area Low-Level Waste Facility at the Savannah River Site
}

\author{
James R. Cook
}

March 20, 2007

Savannah River National Laboratory

Washington Savannah River Company

Savannah River Site

Aiken. SC 29808

Prepared for the U.S. Department of Energy Under

Contract Number DE-AC09-96SR18500

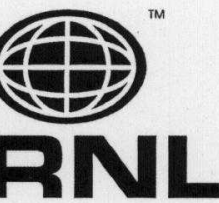




\section{DISCLAIMER}

This report was prepared for the United States Department of Energy under Contract No. DE-AC09-96SR18500 and is an account of work performed under that contract. Neither the United States Department of Energy, nor WSRC, nor any of their employees makes any warranty, expressed or implied, or assumes any legal liability or responsibility for accuracy, completeness, or usefulness, of any information, apparatus, or product or process disclosed herein or represents that its use will not infringe privately owned rights. Reference herein to any specific commercial product, process, or service by trade name, trademark, name, manufacturer or otherwise does not necessarily constitute or imply endorsement, recommendation, or favoring of same by Washington Savannah River Company or by the United States Government or any agency thereof. The views and opinions of the authors expressed herein do not necessarily state or reflect those of the United States Government or any agency thereof.

Printed in the United States of America

Prepared For

U.S. Department of Energy 


\section{Table of Contents}

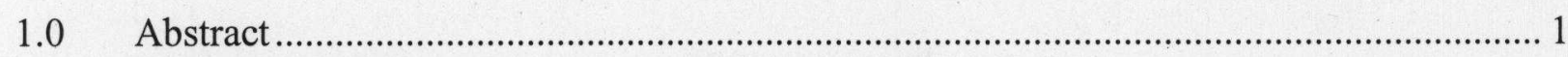

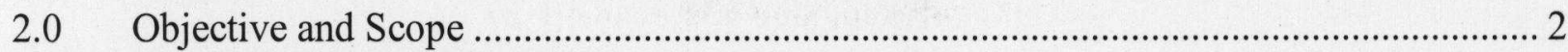

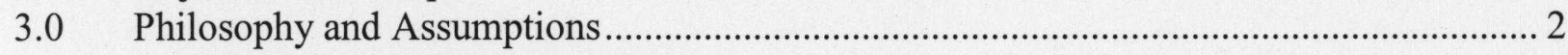

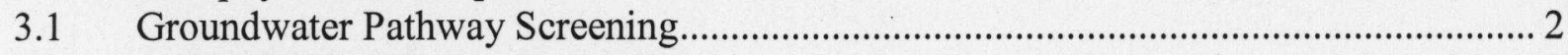

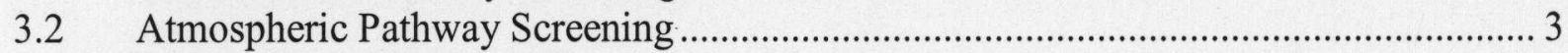

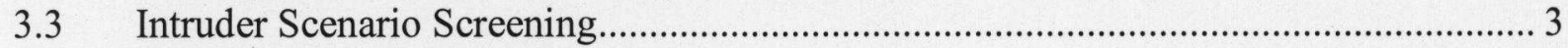

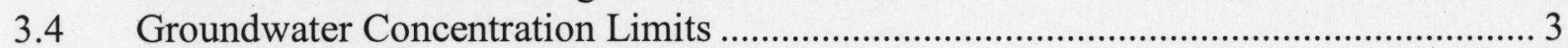

4.0 Regulatory Framework and Data Sources .................................................................... 4

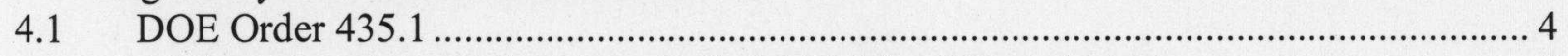

4.2 Environmental Protection Agency Safe Drinking Water Act........................................ 5

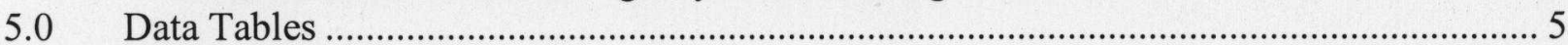

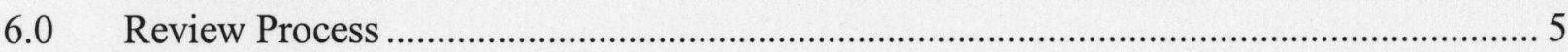

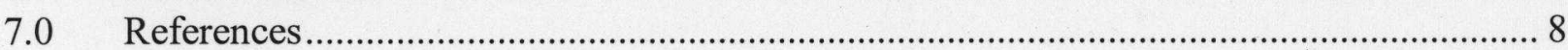


WSRC-STI-2006-00162

Rev. 0

\section{List of Tables}

Table 1 Performance Objectives, Assessment Requirements, and Points of Compliance From DOE Order

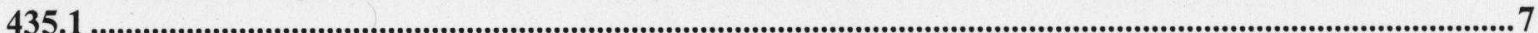


Rev. 0

\section{Acronyms}

PA

SRS
Performance Assessment

Savannah River Site 


\subsection{ABSTRACT}

The Savannah River Site disposes of low-level radioactive waste within on-site engineered disposal facilities. The Savannah River Site must demonstrate that these disposals meet the requirements of DOE Order 435.1 through a process known as performance assessment (PA). The objective of this document is to provide the radionuclide-specific data needed for the PA calculations. This work is part of an on-going program to periodically review and update existing PA work as new data becomes available. Revision of the E-Area Low-Level Waste Facility PA is currently underway.

The number of radionuclides selected to undergo detailed analysis in the PA is determined by a screening process. The basis of this process is described.

Radionuclide-specific data for half-lives, decay modes, daughters, dose conversion factors and groundwater concentration limits are presented with source references and methodologies. 


\subsection{OBJECTIVE AND SCOPE}

The objectives of this document are to explain the following as they relate to performance assessments conducted at the Savannah River Site:

1. the radionuclide screening methodology,

2. the source documents used for radionuclide-specific values for the numerical parameters and justification for their selection,

3. the radionuclide-specific input values for the PA and justification for their selection, and

4. the review process of this document

The scope of this document is to provide radionuclide-specific input values for PA modeling at the Savannah River Site.

\subsection{PHILOSOPHY AND ASSUMPTIONS}

A basic philosophy of the PA at SRS is to use screening processes to reduce the number of radionuclides for which detailed calculations must be performed. This is done using a combination of a conservative, high-level model and site-specific process knowledge for the groundwater and atmospheric pathways as well as for hypothetical intruder scenarios.

Sections 3.1 through 3.3 describe the philosophy and assumptions that are used in the groundwater, atmospheric and intruder pathway screening, respectively. Section 3.4 describes the methodology used to calculate groundwater concentration limits for those radionuclides without a published Safe Drinking Water Act Maximum Contaminant Level.

\subsection{Groundwater Pathway Screening}

The methodology developed by the National Council on Radiological Protection and Measurements (NCRP 1996) described in Section 4.2.2 of Volume 1 of that report was used to reduce the number of radionuclides requiring detailed analysis of the groundwater pathway from 826 to 86 (Taylor and Collard 2005). The list was further reduced to 35 radionuclides by considering only those radionuclides with a total disposed inventory in all E-Area disposal units (Stallings and Swingle 2005) that exceeds the trigger values calculated in Taylor and Collard 2005 (Cook 2006). Trigger values are the inventory of a radionuclide that would give a dose of $0.04 \mathrm{mrem} /$ year using the NCRP screening methodology. The 35 radionuclides are the parent radionuclides used in the groundwater transport calculations. A number of data tables have been included in this report. Table A- 1 lists the 826 radionuclides considered in the PA process. Table A-2 lists the 86 radionuclides remaining after groundwater screening. Table A-3 lists the 35 parent radionuclides that were used in the groundwater transport calculations. 


\subsection{Atmospheric Pathway Screening}

The methodology for reducing the number of radionuclides requiring detailed analysis of the atmospheric pathway relied on the following:

1. Applying the screening methodology developed by the National Council on Radiological Protection and Measurements (NCRP 1996) described in Section 2.2.1 of Volume 1 of that report and calculating trigger values (inventory that would give a dose of 0.1 $\mathrm{mrem} /$ year),

2. Removing from consideration all but those elements and associated radionuclides that could be released from a closed E-Area disposal unit in the vapor phase and

3. Eliminating from consideration those volatile radionuclides that are not currently found in the E-Area Low-Level Waste Facility or were below trigger values calculated in the first screening step.

When this screening process was completed the number of radionuclides requiring detailed analysis of the atmospheric pathway was reduced from 826 to 15 (Crapse and Cook 2006). Table A-4 is a list of these 15 radionuclides.

\subsection{Intruder Scenario Screening}

The methodology developed by the National Council on Radiological Protection and Measurements (NCRP 1996) described in Section 4.2 .3 of Volume 1 of that report was used to reduce the number of radionuclides requiring detailed analysis of intruder scenarios from 826 to 78 parent radionuclides (Cook and Wilhite 2004). These radionuclides were then included in a computer application that calculates intruder-based disposal limits for each disposal unit in EArea (Koffman 2004). Table A-5 lists these 78 parent radionuclides.

\subsection{Groundwater Concentration Limits}

When available, groundwater concentration limits provided by the USEPA as a part of the Safe Drinking Water Act are used (USEPA 1992). For the remainder of the radionuclides, groundwater concentration limits were calculated using the dose conversion factors in Federal Regulatory Guidance 11 (Eckerman 1988), a drinking water consumption rate of 730 liters/year and a dose limit if $4 \mathrm{mrem} / \mathrm{year}$ :

$$
\text { Limit }(\mathrm{pCi} / \mathrm{L})=4 \mathrm{mrem} / \mathrm{yr} /(\mathrm{DCF}(\mathrm{mrem} / \mathrm{pCi}) * 730 \mathrm{~L} / \mathrm{yr})
$$

These are given in Table A- 6 .

\subsection{Abbreviated Radionuclide Decay Chains in Groundwater Analysis}

The ELLWF PA Revision Core team agreed to exclude from the groundwater transport analysis those radioactive daughter isotopes with half-lives less than 5 years. This was done to reduce the number of radionuclides modeled. A detailed analysis was not done; rather, it was judged that neglecting these daughters would not compromise the results of the analysis. The assumption 
was not strictly based on the concept of secular equilibrium, but was a practical judgment to facilitate the transport analysis. The subsequent dose analysis uses all of the daughters.

Using the RadDecay ${ }^{\circledR}$ software, there are a total of 45 radioactive daughters, with half-lives less than five years, from the 35 parent radionuclides that are to be analyzed in the groundwater analysis (Section 3.1). These daughters range in half-life from 1.9 years for Th-228 to 3E-7 seconds for Po-212.

The 35 parent radionuclides were considered to check on the potential significance of daughters with half-lives less than 5 years. Of the 35 parents, 10 (C-14, Cl-36, H-3, I-129, K-40, Nb-94, $\mathrm{Ni}-59$, Pd-107, Se-79, and Tc-99) have daughters that are stable. Two of the parents (Mo-93 and $\mathrm{Zr}-93$ ) decay to $\mathrm{Nb}-93 \mathrm{~m}$, whose half-life is 14.6 years and which subsequently decays to $\mathrm{Nb}-93$, which is stable. The chains for the remaining parents were examined to consider the difference in half-life between the first daughter whose half-life is less than 5 years and its parent. These ratios range from 1.2E-04 for $\mathrm{Cm}-244, \mathrm{Pu}-240$, Th-232 and U-236 to 4.1E-12 for Pu-239 and U235. The ratio of half-lives equals the ratio of the radioactivity (i.e., curies) of the daughter and parent. For those radionuclides whose half-life ratio is $1.2 \mathrm{E}-04$, the short-lived daughters are no more than $0.012 \%$ of the parent's radioactivity. Thus, these and subsequent daughters are judged not to be significant in the transport analysis.

\subsection{REGULATORY FRAMEWORK AND DATA SOURCES 4.1 DOE Order 435.1}

The specific performance objectives for solid waste disposal in E-Area are contained in USDOE Order 435.1 (USDOE 1999):

Performance Objectives. Low-level waste disposal facilities shall be sited, designed, operated, maintained, and closed so that a reasonable expectation exists that the following performance objectives will be met for waste disposed of after September 26, 1988:

(a) Dose to representative members of the public shall not exceed $25 \mathrm{mrem}(0.25 \mathrm{mSv})$ in a year total effective dose equivalent from all exposure pathways, excluding the dose from radon and its progeny in air.

(b) Dose to representative members of the public via the air pathway shall not exceed 10 mrem $(0.10 \mathrm{mSv})$ in a year total effective dose equivalent, excluding the dose from radon and its progeny.

(c) Release of radon shall be less than an average flux of $20 \mathrm{pCi} / \mathrm{m}^{2} / \mathrm{s}\left(0.74 \mathrm{~Bq} / \mathrm{m}^{2} / \mathrm{s}\right)$ at the surface of the disposal facility. Alternatively, a limit of $0.5 \mathrm{pCi} / \mathrm{L}(0.0185 \mathrm{~Bq} / \mathrm{L})$ of air may be applied at the boundary of the facility.

In addition to the performance objectives, the Order requires an assessment of impacts to water resources and to hypothetical persons assumed to inadvertently intrude for a temporary period into the low-level waste disposal facility. Table 1 is a summary of these requirements 


\subsection{Environmental Protection Agency Safe Drinking Water Act}

The guidance for implementation of the Safe Drinking Water Act published by the USEPA is used as the primary source for groundwater concentration limits (USEPA 2002). These consist of published Maximum Contaminant Levels for beta-gamma radionuclides, $15 \mathrm{pCi} / \mathrm{L}$ for alphaemitting radionuclides and $30 \mu \mathrm{g} / \mathrm{L}$ for each uranium isotope.

\subsection{DATA TABLES}

The radionuclide data to be used in the E-Area Performance Assessment conducted in 2006 and 2007 are given in Tables A-1 through A-10 in Appendix A. The PA relies on a consistent application of radionuclide properties throughout the analysis. The data tables gives references for the sources of radionuclide data. In addition to the tables already described, the following are presented in Appendix A. Radionuclide half lives and branching fractions as given in Table A-7, information on whether a transformation is by alpha decay or not for branching fractions greater than or equal to 0.01 , which is needed to determine a groundwater concentration limit, is found in Table A-8, ingestion effective dose conversion factors are shown in three sets of units in Table A-9, the radioactive decay constants are given in Table $\mathrm{A}-10$, and the values of $\mathrm{Moles} / \mathrm{Ci}$ and $\mathrm{g} / \mathrm{Ci}$ for each radionuclide of interest in the groundwater transport calculations (parents and daughters with a half-life greater than 5 years), calculated using the equations

Curies per mole $=($ Avagadro's Number * natural $\log$ of 2$) /($ Radionuclide half life $) /$ (disintegrations per year per Curie)

and

Curies per gram $=$ Curies per mole $/$ grams per mole

are presented in Table A-11.

\subsection{REVIEW PROCESS}

This report provides baseline data being used in the E-Area PA revision. The objective of the review process is not only to improve the quality of this document, but also to enhance its credibility. Performance Assessments must undergo a number of evaluations, including technical reviews from the outside scientific community. The internal review process will be based on the SRNL Technical Report Design Check Guidelines (WSRC-IM-2002-00011, Rev.2)

All review instructions, comments, and manner in which the review comments were addressed will be documented and archived. The process will be taken full cycle, i.e., all comments will be resolved to the reviewers' satisfaction. 
WSRC-STI-2006-00162

Rev. 0 
Table 1 Performance Objectives, Assessment Requirements, and Points of Compliance From DOE Order 435.1

\begin{tabular}{|c|c|c|}
\hline $\begin{array}{l}\text { Performance } \\
\text { Objective }^{1}\end{array}$ & Measure & Point of Compliance \\
\hline All pathways & $\begin{array}{l}\leq 25 \text { mrem in a year, not including } \\
\text { doses from radon and its progeny } \\
\text { in air }\end{array}$ & $\begin{array}{l}\text { Point of highest projected dose } \\
\text { or concentration beyond a } 100- \\
\text { meter buffer zone surrounding } \\
\text { the disposed waste. }\end{array}$ \\
\hline Air pathway & $\begin{array}{l}\leq 10 \text { mrem in a year, not including } \\
\text { doses from radon and progeny }\end{array}$ & $\begin{array}{l}\text { Point of highest projected dose } \\
\text { or concentration beyond a } 100- \\
\text { meter buffer zone surrounding } \\
\text { the disposed waste. }\end{array}$ \\
\hline \multirow[t]{3}{*}{ Radon } & either & \\
\hline & $\begin{array}{l}\text { (1) an average flux of } \\
\leq 20 \mathrm{pCi} / \mathrm{m}^{2} / \mathrm{s} \text {, or }\end{array}$ & Disposal facility surface \\
\hline & $\begin{array}{l}\text { (2) an air concentration of } \\
\leq 0.5 \mathrm{pCi} / \mathrm{L}\end{array}$ & $\begin{array}{l}\text { Point of highest projected dose } \\
\text { or concentration beyond a } 100- \\
\text { meter buffer zone surrounding } \\
\text { the disposed waste. }\end{array}$ \\
\hline $\begin{array}{l}\text { Assessment } \\
\text { Requirement }^{2}\end{array}$ & Measure & Point of Compliance \\
\hline \multirow{2}{*}{$\begin{array}{l}\text { Hypothetical } \\
\text { inadvertent } \\
\text { intruder }\end{array}$} & $\begin{array}{l}100 \text { mrem in a year from chronic } \\
\text { exposure }\end{array}$ & Disposal facility \\
\hline & 500 mrem from a single event & Disposal facility \\
\hline $\begin{array}{l}\text { Impact on Water } \\
\text { Resources }\end{array}$ & $\begin{array}{l}\text { The SRS interpretation is that } \\
\text { concentrations of radioactive } \\
\text { contaminants should not exceed } \\
\text { standards for public drinking water } \\
\text { supplies established by the } \\
\text { USDOE ( } 40 \text { CFR Part } 141) \text {. }\end{array}$ & $\begin{array}{l}\text { Point of highest projected dose } \\
\text { or concentration beyond a } 100 \\
\text { meter buffer zone surrounding } \\
\text { the disposed waste. }\end{array}$ \\
\hline
\end{tabular}

1. USDOE Order 435.1 requires that low-level waste disposal facilities shall be sited, designed, operated, maintained, and closed so that a reasonable expectation exists that the performance objectives will be met for waste disposed of after September 26, 1988.

2. USDOE Order 435.1 also requires that the performance assessment include, for purposes of establishing limits on radionuclides that may be disposed of near-surface, an assessment of the impacts on water resources and an assessment of impacts calculated for a hypothetical person assumed to inadvertently intrude for a temporary period into the low-level waste disposal facility. 


\subsection{REFERENCES}

Cook, James R. 2006. E-mail to Don Sink Short Radionuclide List August 8, 2006.

Cook, James R. and Wilhite, Elmer L. 2004. Special Analysis: Radionuclide Screening Analysis for E-Area. WSRC-TR-2004-00294. Westinghouse Savannah River Company, Aiken SC.

Crapse, Kimberly P. and Cook, James R. 2006. Atmospheric Pathway Screening Analysis for the E-Area Low Level Waste Facility. WSRC-STI-2006-00159, Savannah River National Laboratory, Aiken, SC.

Eckerman, Keith F, Wolbarst, Anthony B. and Richardson, Allan C. B. 1988. Limiting Values of Radionuclide Intake and Air Concentration and Dose Conversion Factors for Inhalation, Submersion, and Ingestion. Federal Guidance Report No. 11. U.S. Environmental Protection Agency, Washington, DC.

ICRP 1979. Limits on Intake of Radionuclides by Workers. International Commission on Radiological Protection Report 30. Pergamon Press, Oxford.

ICRP 1983. Radionuclide Transformations: Energy and Intensity of Emissions. International Commission on Radiological Protection Report 38. Pergamon Press, Oxford.

Koffman, Larry D 2004. An Automated Inadvertent Intruder Analysis Application. WSRCTR-2004-00293, Revision 0. Savannah River National Laboratory, Aiken, SC.

NCRP 1996. Screening Models for Releases of Radionuclides to Atmosphere, Surface Water, and Ground, NCRP Report No. 123, Volumes I and II, National Council on Radiation Protection and Measurements, Bethesda, MD.

Stallings, M. E. and Swingle, R. F. 2005. E-Area Performance Assessment Interim Measures Assessment FY2005. WSRC-RP-2005-01783. River National Laboratory, Aiken, SC.

Taylor, Glenn A. and Collard, Leonard B. 2005. Automated Groundwater Screening. WSRCTR-2005-00203. Savannah River National Laboratory, Aiken, SC.

Tuli, Jagdish K. 2005. Nuclear Wallet Cards. Brookhaven National Laboratory, Upton, NY.

USDOE 1999. Radioactive Waste Management, Order 435.1, U. S. Department of Energy, Washington, DC.

USEPA 2004. National Primary Drinking Water Regulations, 40 CFR Part 141, 8-27-04 Edition. U. S. Environmental Protection Agency, Washington, DC.

USEPA 2002. Implementation Guidance for Radionuclides. EPA-816-F-00-002. U.S. Environmental Protection Agency, Washington, DC. 
WSRC-STI-2006-00162

Rev. 0

\section{APPENDIX A}


Table A-1 Radionuclides to be Considered in the PA Process (ICRP 1983)

\begin{tabular}{|c|c|c|c|c|}
\hline Ac- 223 & As-70 & Bi-202 & $\mathrm{Cd}-117 \mathrm{~m}$ & Co-61 \\
\hline Ac- 224 & As-71 & Bi-203 & $\mathrm{Ce}-134$ & $\mathrm{Co}-62 \mathrm{~m}$ \\
\hline Ac- 225 & As-72 & Bi-205 & $\mathrm{Ce}-135$ & $\mathrm{Cr}-48$ \\
\hline Ac- 226 & As-73 & Bi-206 & Ce-137 & $\mathrm{Cr}-49$ \\
\hline Ac- 227 & As-74 & Bi-207 & $\mathrm{Ce}-137 \mathrm{~m}$ & Cr-51 \\
\hline Ac- 228 & As-76 & $\mathrm{Bi}-210$ & Ce-139 & Cs-125 \\
\hline Ag-102 & As-77 & $\mathrm{Bi}-210 \mathrm{~m}$ & Ce-141 & Cs-126 \\
\hline Ag-103 & As-78 & Bi-211 & Ce-143 & Cs-127 \\
\hline Ag-104 & At-207 & $\mathrm{Bi}-212$ & $\mathrm{Ce}-144$ & Cs-128 \\
\hline Ag-104m & At-211 & Bi-213 & Cf-244 & Cs-129 \\
\hline Ag-105 & At-215 & Bi-214 & Cf-246 & Cs-130 \\
\hline Ag-106 & At-216 & Bk-245 & Cf-248 & Cs-131 \\
\hline Ag-106m & At-217 & Bk-246 & Cf-249 & Cs-132 \\
\hline Ag-108 & At- 218 & Bk-247 & Cf-250 & Cs-134 \\
\hline Ag-108m & Au-193 & Bk-249 & Cf-251 & $\mathrm{Cs}-134 \mathrm{~m}$ \\
\hline Ag-109m & Au-194 & $\mathrm{Bk}-250$ & $\mathrm{Cf}-252$ & $\mathrm{Cs}-135$ \\
\hline Ag-110 & $\mathrm{Au}-195$ & $\mathrm{Br}-74$ & Cf-253 & $\mathrm{Cs}-135 \mathrm{~m}$ \\
\hline $\mathrm{Ag}-110 \mathrm{~m}$ & $\mathrm{Au}-195 \mathrm{~m}$ & $\mathrm{Br}-74 \mathrm{~m}$ & Cf-254 & Cs-136 \\
\hline Ag-111 & $\mathrm{Au}-198$ & $\mathrm{Br}-75$ & $\mathrm{Cl}-36$ & Cs-137 \\
\hline Ag-112 & $\mathrm{Au}-198 \mathrm{~m}$ & $\mathrm{Br}-76$ & $\mathrm{Cl}-38$ & Cs-138 \\
\hline Ag-115 & Au-199 & $\mathrm{Br}-77$ & $\mathrm{Cl}-39$ & $\mathrm{Cu}-60$ \\
\hline Al-26 & $\mathrm{Au}-200$ & $\mathrm{Br}-80$ & $\mathrm{Cm}-238$ & $\mathrm{Cu}-61$ \\
\hline Al-28 & $\mathrm{Au}-200 \mathrm{~m}$ & $\mathrm{Br}-80 \mathrm{~m}$ & $\mathrm{Cm}-240$ & $\mathrm{Cu}-62$ \\
\hline Am-237 & $\mathrm{Au}-201$ & $\mathrm{Br}-82$ & $\mathrm{Cm}-241$ & $\mathrm{Cu}-64$ \\
\hline Am-238 & Ba-126 & $\mathrm{Br}-83$ & $\mathrm{Cm}-242$ & $\mathrm{Cu}-66$ \\
\hline Am-239 & Ba-128 & $\mathrm{Br}-84$ & $\mathrm{Cm}-243$ & $\mathrm{Cu}-67$ \\
\hline Am-240 & $\mathrm{Ba}-131$ & C-11 & $\mathrm{Cm}-244$ & Dy-155 \\
\hline Am-241 & Ba-131m & C-14 & $\mathrm{Cm}-245$ & Dy-157 \\
\hline Am-242 & Ba-133 & $\mathrm{Ca}-41$ & $\mathrm{Cm}-246$ & Dy-159 \\
\hline Am-242m & Ba-133m & $\mathrm{Ca}-45$ & $\mathrm{Cm}-247$ & Dy-165 \\
\hline Am-243 & Ba-135m & $\mathrm{Ca}-47$ & $\mathrm{Cm}-248$ & Dy-166 \\
\hline Am-244 & Ba-137m & $\mathrm{Ca}-49$ & $\mathrm{Cm}-249$ & Er-161 \\
\hline Am-244m & Ba-139 & Cd-104 & $\mathrm{Cm}-250$ & Er-165 \\
\hline Am-245 & Ba-140 & $\mathrm{Cd}-107$ & Co-55 & Er-169 \\
\hline Am-246 & Ba-141 & Cd-109 & Co-56 & Er-171 \\
\hline Am-246m & Ba-142 & $\mathrm{Cd}-113$ & Co-57 & Er-172 \\
\hline Ar-37 & Be-10 & $\mathrm{Cd}-113 \mathrm{~m}$ & $\mathrm{Co}-58$ & Es-250 \\
\hline Ar-39 & $\mathrm{Be}-7$ & Cd-115 & $\mathrm{Co}-58 \mathrm{~m}$ & Es-251 \\
\hline Ar-41 & $\mathrm{Bi}-200$ & $\mathrm{Cd}-115 \mathrm{~m}$ & $\mathrm{Co}-60$ & Es-253 \\
\hline As-69 & Bi-201 & Cd-117 & $\mathrm{Co}-60 \mathrm{~m}$ & Es-254 \\
\hline
\end{tabular}


Table A-1 Radionuclides to be Considered in the PA Process (ICRP 1983)

\begin{tabular}{|c|c|c|c|c|}
\hline Es- $254 \mathrm{~m}$ & Gd-148 & Но-159 & In-119m & La-137 \\
\hline Eu-145 & Gd-149 & Ho-161 & Ir-182 & La-138 \\
\hline Eu-146 & Gd-151 & Но-162 & Ir-184 & La-140 \\
\hline Eu-147 & Gd-152 & Ho- $162 \mathrm{~m}$ & Ir-185 & La-141 \\
\hline Eu-148 & Gd-153 & Но-164 & Ir-186a & La-142 \\
\hline Eu-149 & Gd-159 & Ho- $164 \mathrm{~m}$ & Ir-186b & La-143 \\
\hline Eu-150a & Ge-66 & Но-166 & Ir-187 & Lu-169 \\
\hline Eu-150b & Ge-67 & Ho-166m & Ir-188 & Lu-170 \\
\hline Eu-152 & Ge-68 & Но-167 & Ir-189 & Lu-171 \\
\hline Eu-152m & Ge-69 & I- 120 & Ir-190 & Lu-172 \\
\hline Eu-154 & Ge-71 & $\mathrm{I}-120 \mathrm{~m}$ & Ir- $190 \mathrm{~m}$ & Lu-173 \\
\hline Eu-155 & Ge-75 & $\mathrm{I}-121$ & Ir-190n & Lu-174 \\
\hline Eu-156 & Ge-77 & I-122 & Ir-191m & Lu-174m \\
\hline Eu-157 & $\mathrm{Ge}-78$ & I-123 & Ir-192 & Lu-176 \\
\hline Eu-158 & $\mathrm{H}-3$ & I-124 & Ir-192m & Lu-176m \\
\hline F-18 & Hf-170 & $\mathrm{I}-125$ & Ir-194 & Lu-177 \\
\hline $\mathrm{Fe}-52$ & Hf-172 & I-126 & Ir-194m & Lu-177m \\
\hline $\mathrm{Fe}-55$ & Hf-173 & $\mathrm{I}-128$ & Ir-195 & Lu-178 \\
\hline Fe-59 & Hf-174 & I-129 & Ir-195m & Lu-178m \\
\hline $\mathrm{Fe}-60$ & Hf-175 & I-130 & $\mathrm{K}-38$ & Lu-179 \\
\hline Fm-252 & $\mathrm{Hf}-177 \mathrm{~m}$ & I-131 & $\mathrm{K}-40$ & Md-257 \\
\hline Fm-253 & Hf- $178 m$ & $\mathrm{I}-132$ & $\mathrm{~K}-42$ & Md-258 \\
\hline Fm-254 & Hf $-179 m$ & $\mathrm{I}-132 \mathrm{~m}$ & $\mathrm{~K}-43$ & $\mathrm{Mg}-28$ \\
\hline Fm-255 & $\mathrm{Hf}-180 \mathrm{~m}$ & I-133 & $\mathrm{K}-44$ & $\mathrm{Mn}-51$ \\
\hline Fm-257 & Hf-181 & I-134 & $K-45$ & $\mathrm{Mn}-52$ \\
\hline Fr-219 & Hf-182 & I-135 & $\mathrm{Kr}-74$ & $\mathrm{Mn}-52 \mathrm{~m}$ \\
\hline Fr-220 & $\mathrm{Hf}-182 \mathrm{~m}$ & In-109 & $\mathrm{Kr}-76$ & $\mathrm{Mn}-53$ \\
\hline Fr-221 & Hf-183 & In-110a & $\mathrm{Kr}-77$ & $\mathrm{Mn}-54$ \\
\hline Fr-222 & Hf-184 & In- $110 b$ & $\mathrm{Kr}-79$ & $\mathrm{Mn}-56$ \\
\hline Fr-223 & $\mathrm{Hg}-193$ & In-111 & $\mathrm{Kr}-81$ & Mo-101 \\
\hline Ga-65 & $\mathrm{Hg}-193 \mathrm{~m}$ & In-112 & $\mathrm{Kr}-81 \mathrm{~m}$ & Mo-90 \\
\hline Ga-66 & $\mathrm{Hg}-194$ & In- $113 m$ & $\mathrm{Kr}-83 \mathrm{~m}$ & Mo-93 \\
\hline Ga-67 & $\mathrm{Hg}-195$ & In-114 & $\mathrm{Kr}-85$ & Mo-93m \\
\hline Ga-68 & $\mathrm{Hg}-195 \mathrm{~m}$ & In- $114 m$ & $\mathrm{Kr}-85 \mathrm{~m}$ & Мo-99 \\
\hline $\mathrm{Ga}-70$ & Hg-197 & In-115 & $\mathrm{Kr}-87$ & N-13 \\
\hline Ga-72 & $\mathrm{Hg}-197 \mathrm{~m}$ & In- $115 \mathrm{~m}$ & $\mathrm{Kr}-88$ & $\mathrm{Na}-22$ \\
\hline $\mathrm{Ga}-73$ & $\mathrm{Hg}-199 \mathrm{~m}$ & In-116m & La-131 & $\mathrm{Na}-24$ \\
\hline Gd-145 & $\mathrm{Hg}-203$ & In-117 & La-132 & $\mathrm{Nb}-88$ \\
\hline Gd-146 & Ho-155 & In- $117 \mathrm{~m}$ & La-134 & $\mathrm{Nb}-89 \mathrm{a}$ \\
\hline Gd-147 & Но-157 & In-119 & La-135 & $\mathrm{Nb}-89 \mathrm{~b}$ \\
\hline
\end{tabular}


Table A-1 Radionuclides to be Considered in the PA Process (ICRP 1983)

\begin{tabular}{|c|c|c|c|c|}
\hline $\mathrm{Nb}-90$ & Os-185 & Pm-144 & Pt-197m & $\mathrm{Re}-182 \mathrm{~b}$ \\
\hline $\mathrm{Nb}-93 \mathrm{~m}$ & Os-189m & Pm-145 & Pt-199 & Re-184 \\
\hline $\mathrm{Nb}-94$ & Os-190m & Pm-146 & Pt-200 & $\mathrm{Re}-184 \mathrm{~m}$ \\
\hline $\mathrm{Nb}-95$ & Os-191m & Pm-147 & $\mathrm{Pu}-234$ & Re-186 \\
\hline $\mathrm{Nb}-95 \mathrm{~m}$ & Os-191 & Pm-148 & $\mathrm{Pu}-235$ & $\mathrm{Re}-186 \mathrm{~m}$ \\
\hline $\mathrm{Nb}-96$ & Os-193 & Pm-148m & $\mathrm{Pu}-236$ & Re-187 \\
\hline $\mathrm{Nb}-97$ & Os-194 & Pm-149 & $\mathrm{Pu}-237$ & Re-188 \\
\hline $\mathrm{Nb}-97 \mathrm{~m}$ & P-30 & Pm-150 & $\mathrm{Pu}-238$ & $\mathrm{Re}-188 \mathrm{~m}$ \\
\hline $\mathrm{Nb}-98$ & P-32 & Pm-151 & Pu-239 & Re-189 \\
\hline Nd-136 & $P-33$ & Po-203 & $\mathrm{Pu}-240$ & Rh-100 \\
\hline Nd-138 & $\mathrm{Pa}-227$ & Рo-205 & $\mathrm{Pu}-241$ & $\mathrm{Rh}-101$ \\
\hline Nd-139 & $\mathrm{Pa}-228$ & Рo-207 & $\mathrm{Pu}-242$ & $\mathrm{Rh}-101 \mathrm{~m}$ \\
\hline $\mathrm{Nd}-139 \mathrm{~m}$ & $\mathrm{~Pa}-230$ & Рo-210 & $\mathrm{Pu}-243$ & $\mathrm{Rh}-102$ \\
\hline Nd-141 & $\mathrm{Pa}-231$ & Po-211 & $\mathrm{Pu}-244$ & $\mathrm{Rh}-102 \mathrm{~m}$ \\
\hline $\mathrm{Nd}-141 \mathrm{~m}$ & $\mathrm{~Pa}-232$ & Po-212 & $\mathrm{Pu}-245$ & $\mathrm{Rh}-103 \mathrm{~m}$ \\
\hline Nd-147 & $\mathrm{Pa}-233$ & Рo-213 & $\mathrm{Pu}-246$ & Rh-105 \\
\hline Nd-149 & $\mathrm{Pa}-234$ & Рo-214 & Ra-222 & Rh-106 \\
\hline Nd-151 & $\mathrm{Pa}-234 \mathrm{~m}$ & Po-215 & Ra-223 & $\mathrm{Rh}-106 \mathrm{~m}$ \\
\hline $\mathrm{Ne}-19$ & $\mathrm{~Pb}-195 \mathrm{~m}$ & Рo-216 & Ra-224 & Rh-107 \\
\hline $\mathrm{Ni}-56$ & $\mathrm{~Pb}-198$ & Рo-218 & Ra-225 & Rh-99 \\
\hline $\mathrm{Ni}-57$ & $\mathrm{~Pb}-199$ & $\operatorname{Pr}-136$ & Ra-226 & Rh-99m \\
\hline $\mathrm{Ni}-59$ & $\mathrm{~Pb}-200$ & Pr-137 & Ra-227 & $\mathrm{Rn}-218$ \\
\hline $\mathrm{Ni}-63$ & $\mathrm{~Pb}-201$ & Pr-138 & Ra-228 & $R n-219$ \\
\hline $\mathrm{Ni}-65$ & $\mathrm{~Pb}-202$ & $\operatorname{Pr}-138 \mathrm{~m}$ & $\mathrm{Rb}-79$ & $\mathrm{Rn}-220$ \\
\hline $\mathrm{Ni}-66$ & $\mathrm{~Pb}-202 \mathrm{~m}$ & Pr-139 & $\mathrm{Rb}-80$ & $\mathrm{Rn}-222$ \\
\hline $\mathrm{Np}-232$ & $\mathrm{~Pb}-203$ & Pr-142 & $\mathrm{Rb}-81$ & Ru-103 \\
\hline Np-233 & $\mathrm{Pb}-205$ & $\operatorname{Pr}-142 \mathrm{~m}$ & $\mathrm{Rb}-81 \mathrm{~m}$ & Ru-105 \\
\hline $\mathrm{Np}-234$ & $\mathrm{~Pb}-209$ & Pr-143 & $\mathrm{Rb}-82$ & Ru-106 \\
\hline $\mathrm{Np}-235$ & $\mathrm{~Pb}-210$ & Pr-144 & $\mathrm{Rb}-82 \mathrm{~m}$ & Ru-94 \\
\hline $\mathrm{Np}-236 \mathrm{a}$ & $\mathrm{Pb}-211$ & $\operatorname{Pr}-144 \mathrm{~m}$ & $\mathrm{Rb}-83$ & Ru-97 \\
\hline $\mathrm{Np}-236 \mathrm{~b}$ & $\mathrm{~Pb}-212$ & Pr-145 & $\mathrm{Rb}-84$ & S-35 \\
\hline Np-237 & $\mathrm{Pb}-214$ & Pr-147 & $\mathrm{Rb}-86$ & Sb-115 \\
\hline Np-238 & Pd-100 & Pt-186 & $\mathrm{Rb}-87$ & $\mathrm{Sb}-116$ \\
\hline $\mathrm{Np}-239$ & Pd-101 & Pt-188 & $\mathrm{Rb}-88$ & $\mathrm{Sb}-116 \mathrm{~m}$ \\
\hline $\mathrm{Np}-240$ & Pd-103 & Pt-189 & $\mathrm{Rb}-89$ & Sb-117 \\
\hline $\mathrm{Np}-240 \mathrm{~m}$ & Pd-107 & Pt-191 & Re-177 & $\mathrm{Sb}-118 \mathrm{~m}$ \\
\hline O-15 & Pd-109 & Pt-193 & Re-178 & $\mathrm{Sb}-119$ \\
\hline Os-180 & Pm-141 & Pt-193m & Re-180 & $\mathrm{Sb}-120 \mathrm{a}$ \\
\hline Os-181 & Pm-142 & Pt-195m & Re-181 & $\mathrm{Sb}-120 \mathrm{~b}$ \\
\hline Os-182 & Pm-143 & Pt-197 & $\mathrm{Re}-182 \mathrm{a}$ & $\mathrm{Sb}-122$ \\
\hline
\end{tabular}


Table A-1 Radionuclides to be Considered in the PA Process (ICRP 1983)

\begin{tabular}{|c|c|c|c|c|}
\hline Sb-124 & Sn-110 & Та-186 & Te-131 & U-231 \\
\hline $\mathrm{Sb}-124 \mathrm{~m}$ & Sn-111 & Tb-147 & Te-131m & U-232 \\
\hline $\mathrm{Sb}-124 \mathrm{n}$ & Sn-113 & Tb-149 & Te-132 & U-233 \\
\hline $\mathrm{Sb}-125$ & $\mathrm{Sn}-117 \mathrm{~m}$ & $\mathrm{~Tb}-150$ & Te-133 & U-234 \\
\hline $\mathrm{Sb}-126$ & $\mathrm{Sn}-119 \mathrm{~m}$ & Tb-151 & Te-133m & U-235 \\
\hline $\mathrm{Sb}-126 \mathrm{~m}$ & Sn-121 & Tb-153 & Te-134 & U-236 \\
\hline $\mathrm{Sb}-127$ & Sn-121m & Tb-154 & Th-226 & U-237 \\
\hline $\mathrm{Sb}-128 \mathrm{a}$ & Sn-123 & Tb-155 & Th-227 & U-238 \\
\hline $\mathrm{Sb}-128 \mathrm{~b}$ & $\mathrm{Sn}-123 \mathrm{~m}$ & $\mathrm{~Tb}-156 \mathrm{~m}$ & Th-228 & U-239 \\
\hline $\mathrm{Sb}-129$ & Sn-125 & Tb-156n & Th-229 & U-240 \\
\hline $\mathrm{Sb}-130$ & Sn-126 & Tb-157 & Th-230 & V-47 \\
\hline $\mathrm{Sb}-131$ & Sn-127 & $\mathrm{Tb}-158$ & Th-231 & $V-48$ \\
\hline Sc-43 & Sn-128 & $\mathrm{Tb}-160$ & Th-232 & $V-49$ \\
\hline $\mathrm{Sc}-44$ & Sr-80 & Tb-161 & Th-234 & W-176 \\
\hline $\mathrm{Sc}-44 \mathrm{~m}$ & Sr-81 & $\mathrm{Tb}-156$ & Ti-44 & W-177 \\
\hline Sc-46 & $\mathrm{Sr}-82$ & Tc-101 & Ti-45 & W-178 \\
\hline $\mathrm{Sc}-47$ & Sr-83 & Tc-104 & Tl-194 & W-179 \\
\hline Sc-48 & Sr-85 & Тc-93 & Tl-194m & W-181 \\
\hline Sc-49 & $\mathrm{Sr}-85 \mathrm{~m}$ & Tc-93m & Tl-195 & W-185 \\
\hline Se-70 & $\mathrm{Sr}-87 \mathrm{~m}$ & Tc-94 & Tl-197 & W-187 \\
\hline Se-73 & Sr-89 & Tc-94m & Tl-198 & W-188 \\
\hline $\mathrm{Se}-73 \mathrm{~m}$ & Sr-90 & Tc-95 & Tl-198m & Xe-120 \\
\hline Se-75 & Sr-91 & Tc-95m & Tl-199 & $\mathrm{Xe}-121$ \\
\hline $\mathrm{Se}-77 \mathrm{~m}$ & Sr-92 & Тc-96 & Tl-200 & $\mathrm{Xe}-122$ \\
\hline Se-79 & Тa-172 & Tc-96m & T1-201 & $\mathrm{Xe}-123$ \\
\hline Se-81 & Та-173 & Tc-97 & Tl-202 & Xe-125 \\
\hline $\mathrm{Se}-81 \mathrm{~m}$ & Та-174 & Tc-97m & Tl-204 & $\mathrm{Xe}-127$ \\
\hline Se-83 & Тa-175 & Tc-98 & Tl-206 & $\mathrm{Xe}-129 \mathrm{~m}$ \\
\hline $\mathrm{Si}-31$ & Та-176 & Tc-99 & Tl-207 & $\mathrm{Xe}-131 \mathrm{~m}$ \\
\hline $\mathrm{Si}-32$ & Тa-177 & Tc-99m & Tl-208 & $\mathrm{Xe}-133$ \\
\hline Sm-141 & Тa-178a & Te-116 & Tl-209 & $\mathrm{Xe}-133 \mathrm{~m}$ \\
\hline Sm-141m & Ta-178b & Te-121 & Tm-162 & Xe-135 \\
\hline Sm-142 & Та-179 & $\mathrm{Te}-121 \mathrm{~m}$ & Tm-166 & $\mathrm{Xe}-135 \mathrm{~m}$ \\
\hline Sm-145 & Тa-180 & Te-123 & Tm-167 & Xe-138 \\
\hline Sm-146 & Ta-180m & $\mathrm{Te}-123 \mathrm{~m}$ & Tm-170 & Y-86 \\
\hline Sm-147 & Тa-182 & Te-125m & Tm-171 & Y-86m \\
\hline Sm-151 & Ta-182m & Te-127 & Tm-172 & Y-87 \\
\hline Sm-153 & Тa-183 & Te-127m & Tm-173 & Y-88 \\
\hline Sm-155 & Та-184 & Te-129 & Tm-175 & $Y-90$ \\
\hline Sm-156 & Тa-185 & Te-129m & U-230 & $\mathrm{Y}-90 \mathrm{~m}$ \\
\hline
\end{tabular}


Table A-1 Radionuclides to be Considered in the PA Process (ICRP 1983)
Y-91
$\mathrm{Y}-91 \mathrm{~m}$
Y-92
Y-93
Y-94
Y-95
Yb-162
Yb-166
Yb-167
Yb-169
$\mathrm{Yb}-175$
Yb-177
Yb-178
Zn-62
Zn-63
$\mathrm{Zn}-65$
Zn-69
$\mathrm{Zn}-69 \mathrm{~m}$
$\mathrm{Zn}-71 \mathrm{~m}$
$\mathrm{Zn}-72$
Zr-86
Zr- 88
Zr-89
Zr-93
Zr-95
Zr-97 
Table A-2 Radionuclides Remaining after Groundwater Screening (Taylor and Collard 2005)

$\begin{array}{ll}\text { Ag-108m } & \text { Ni-59 } \\ \text { Al-26 } & \text { Np-236a } \\ \text { Am-237 } & \text { Np-237 } \\ \text { Am-241 } & \text { Pa-230 } \\ \text { Am-243 } & \text { Pa-231 } \\ \text { Be-10 } & \text { Pb-202 } \\ \text { Bi-210m } & \text { Pb-205 } \\ \text { Bk-247 } & \text { Pd-107 } \\ \text { Bk-249 } & \text { Pt-193 } \\ \text { C-14 } & \text { Pu-237 } \\ \text { Ca-41 } & \text { Pu-238 } \\ \text { Cd-113 } & \text { Pu-239 } \\ \text { Cf-249 } & \text { Pu-240 } \\ \text { Cf-251 } & \text { Pu-241 } \\ \text { Cf-252 } & \text { Pu-242 } \\ \text { Cl-36 } & \text { Pu-244 } \\ \text { Cm-241 } & \text { Ra-226 } \\ \text { Cm-242 } & \text { Rb-87 } \\ \text { Cm-244 } & \text { Re-186m } \\ \text { Cm-245 } & \text { Re-187 } \\ \text { Cm-246 } & \text { Ru-97 } \\ \text { Cm-247 } & \text { Se-79 } \\ \text { Cm-248 } & \text { Si-32 } \\ \text { Cm-250 } & \text { Sm-146 } \\ \text { Cs-135 } & \text { Sm-147 } \\ \text { Es-253 } & \text { Sn-126 } \\ \text { Fe-60 } & \text { Sr-90 } \\ \text { Gd-152 } & \text { Ta-180 } \\ \text { Ge-68 } & \text { Tc-97 } \\ \text { H-3 } & \text { Tc-98 } \\ \text { Hf-182 } & \text { Tc-99 } \\ \text { Hg-194 } & \text { Te-123 } \\ \text { Ho-166m } & \text { Th-229 } \\ \text { I-129 } & \text { Th-230 } \\ \text { In-115 } & \text { Ti-44 } \\ \text { Ir-192m } & \text { U-233 } \\ \text { K-40 } & \text { U-234 } \\ \text { La-137 } & \text { U-235 } \\ \text { La-138 } & \text { U-236 } \\ \text { Lu-176 } & \text { Zr- }-93 \\ \text { Mn-53 } & \\ \text { Mo-93 } & \\ \text { Nb-94 } & \end{array}$


Rev. 0

Table A-3 35 Parent Radionuclides to be Considered in EArea PA Groundwater Analysis (Cook 2006)
Am-241
Am-243
C-14
Cl-36
$\mathrm{Cm}-244$
$\mathrm{Cm}-245$
$\mathrm{Cm}-247$
$\mathrm{Cm}-248$
H-3
I-129
$\mathrm{K}-40$
Mo-93
$\mathrm{Nb}-94$
Ni-59
$\mathrm{Np}-237$
Pd-107
Pu-238
Pu-239
Pu-240
Pu-241
Pu-242
$\mathrm{Pu}-244$
Ra-226
Se-79
Sn-126
Sr-90
Tc-99
Th-230
Th-232
U-233
U-234
U-235
U-236
U-238
Zr-93 
Table A-4 Radionuclides Remaining after Atmospheric Screening (Crapse and Cook 2006)

C-14

Cl-36

H-3

I-129

S-35

$\mathrm{Sb}-124$

$\mathrm{Sb}-125$

Se-75

Se-79

Sn-113

Sn-119m

Sn-121

Sn-121m

Sn-123

Sn-126 
Table A-5 Radionuclides to be Considered in the Intruder Analysis (Koffman 2004)

\begin{tabular}{|c|c|}
\hline Ac-227 & $\mathrm{Np}-237$ \\
\hline Ag-108m & $\mathrm{Pa}-231$ \\
\hline Al-26 & $\mathrm{Pb}-210$ \\
\hline Am-241 & Pd-107 \\
\hline Am-242m & $\mathrm{Pu}-238$ \\
\hline Am-243 & Pu-239 \\
\hline Ar-39 & $\mathrm{Pu}-240$ \\
\hline Ba-133 & $\mathrm{Pu}-241$ \\
\hline $\mathrm{Bi}-207$ & Pu-242 \\
\hline Bk-249 & $\mathrm{Pu}-244$ \\
\hline C-14 & Ra-226 \\
\hline $\mathrm{Ca}-41$ & Ra-228 \\
\hline $\mathrm{Cd}-113 \mathrm{~m}$ & $\mathrm{Rb}-87$ \\
\hline Cf-249 & S-35 \\
\hline Cf- 250 & $\mathrm{Sb}-125$ \\
\hline Cf-251 & Sc-46 \\
\hline Cf-252 & Se-79 \\
\hline $\mathrm{Cl}-36$ & Sm-151 \\
\hline $\mathrm{Cm}-242$ & $\mathrm{Sn}-121 \mathrm{~m}$ \\
\hline $\mathrm{Cm}-243$ & $\mathrm{Sn}-126$ \\
\hline $\mathrm{Cm}-244$ & Sr-90 \\
\hline $\mathrm{Cm}-245$ & Tc-99 \\
\hline $\mathrm{Cm}-246$ & Th-228 \\
\hline $\mathrm{Cm}-247$ & Th-229 \\
\hline $\mathrm{Cm}-248$ & Th-230 \\
\hline $\mathrm{Co}-60$ & Th-232 \\
\hline Cs-134 & U-232 \\
\hline Cs-135 & U-233 \\
\hline Cs-137 & U-234 \\
\hline Eu-152 & U-235 \\
\hline Eu-154 & U-236 \\
\hline Eu-155 & U-238 \\
\hline $\mathrm{H}-3$ & W-181 \\
\hline I-129 & W-185 \\
\hline$K-40$ & W-188 \\
\hline $\mathrm{Kr}-85$ & Zr-93 \\
\hline \multicolumn{2}{|l|}{ Mo-93 } \\
\hline \multicolumn{2}{|l|}{$\mathrm{Na}-22$} \\
\hline \multicolumn{2}{|l|}{$\mathrm{Nb}-93 \mathrm{~m}$} \\
\hline \multicolumn{2}{|l|}{$\mathrm{Nb}-94$} \\
\hline \multicolumn{2}{|l|}{$\mathrm{Ni}-59$} \\
\hline $\mathrm{Ni}-63$ & \\
\hline
\end{tabular}


Table A-6 Groundwater Concentration Limits (pCi/L) (EPA 2002 and Eckerman 1988)

\begin{tabular}{|c|c|c|}
\hline Radionuclide & Primary Decay Mode & Secondary Decay Mode \\
\hline Ac-225 & 15 & \\
\hline Ac-227 & $3.9 \mathrm{E}-01$ & \\
\hline Ac-228 & $2.5 \mathrm{E}+03$ & \\
\hline Ag-108 & & \\
\hline $\mathrm{Ag}-108 \mathrm{~m}$ & $7.2 \mathrm{E}+02$ & \\
\hline Al-26 & $3.8 \mathrm{E}+02$ & \\
\hline Am-237 & $8.3 \mathrm{E}+04$ & \\
\hline Am-241 & 15 & \\
\hline Am-243 & 15 & \\
\hline Am-245 & $3.0 \mathrm{E}+04$ & \\
\hline Am-246m & $5.8 \mathrm{E}+04$ & \\
\hline At-217 & 15 & \\
\hline At-218 & 15 & \\
\hline Au-194 & $2.9 \mathrm{E}+03$ & \\
\hline $\mathrm{Be}-10$ & $1.2 \mathrm{E}+03$ & \\
\hline Bi-210 & $8.6 \mathrm{E}+02$ & \\
\hline $\mathrm{Bi}-210 \mathrm{~m}$ & 15 & \\
\hline Bi-211 & 15 & \\
\hline Bi-212 & $5.2 \mathrm{E}+03$ & \\
\hline Bi-213 & $7.6 \mathrm{E}+03$ & \\
\hline Bi-214 & $1.9 \mathrm{E}+04$ & \\
\hline Bk-247 & 15 & \\
\hline Bk-249 & 2000 & \\
\hline Bk-250 & $9.4 \mathrm{E}+03$ & \\
\hline C-14 & 2000 & \\
\hline $\mathrm{Ca}-41$ & $4.3 \mathrm{E}+03$ & \\
\hline Cd-113 & $3.2 \mathrm{E}+01$ & \\
\hline Cf-249 & 15 & \\
\hline Cf-250 & 15 & \\
\hline Cf-251 & 15 & \\
\hline Cf-252 & 15 & \\
\hline $\mathrm{Cl}-36$ & 700 & \\
\hline $\mathrm{Cm}-241$ & $1.2 \mathrm{E}+03$ & \\
\hline $\mathrm{Cm}-242$ & 15 & \\
\hline $\mathrm{Cm}-244$ & 15 & \\
\hline $\mathrm{Cm}-245$ & 15 & \\
\hline $\mathrm{Cm}-246$ & 15 & \\
\hline $\mathrm{Cm}-247$ & 15 & \\
\hline $\mathrm{Cm}-248$ & 15 & \\
\hline $\mathrm{Cm}-250$ & 7.1E-02 & 15 \\
\hline Co-60 & 100 & \\
\hline
\end{tabular}


Table A-6 Groundwater Concentration Limits (pCi/L) (EPA 2002 and Eckerman 1988)

\begin{tabular}{|c|c|c|}
\hline Radionuclide & Primary Decay Mode & Secondary Decay Mode \\
\hline Co-60m & $1.5 \mathrm{E}+06$ & \\
\hline Cs-135 & 900 & \\
\hline Es- 253 & 15 & \\
\hline $\mathrm{Fe}-60$ & $3.6 \mathrm{E}+01$ & \\
\hline Fr-221 & 15 & \\
\hline Fr-223 & $6.4 \mathrm{E}+02$ & \\
\hline Ga-68 & $1.6 \mathrm{E}+04$ & \\
\hline Gd-152 & 15 & \\
\hline Ge-68 & $5.1 \mathrm{E}+03$ & \\
\hline $\mathrm{H}-3$ & 20000 & \\
\hline Hf-182 & $3.5 \mathrm{E}+02$ & \\
\hline $\mathrm{Hg}-194$ & $8.9 \mathrm{E}+02$ & \\
\hline Ho-166m & $6.8 \mathrm{E}+02$ & \\
\hline I-129 & 1 & \\
\hline In-115 & 300 & \\
\hline Ir-192 & 100 & \\
\hline Ir-192m & $3.5 \mathrm{E}+03$ & \\
\hline $\mathrm{K}-40$ & $3.0 \mathrm{E}+02$ & \\
\hline La-137 & $1.2 \mathrm{E}+04$ & \\
\hline La-138 & $9.3 \mathrm{E}+02$ & \\
\hline Lu-176 & $7.5 \mathrm{E}+02$ & \\
\hline $\mathrm{Mn}-53$ & $5.1 \mathrm{E}+04$ & \\
\hline Mo-93 & $4.1 \mathrm{E}+03$ & \\
\hline $\mathrm{Nb}-93 \mathrm{~m}$ & 1000 & \\
\hline Nb-94 & $7.7 \mathrm{E}+02$ & \\
\hline $\mathrm{Ni}-59$ & 300 & \\
\hline Np-233 & $7.4 \mathrm{E}+05$ & \\
\hline Np-236a & $6.3 \mathrm{E}+00$ & \\
\hline Np-237 & 15 & \\
\hline Np-239 & 300 & \\
\hline \multicolumn{3}{|l|}{$\mathrm{Np}-240 \mathrm{~m}$} \\
\hline P-32 & 30 & \\
\hline $\mathrm{Pa}-230$ & 600 & 15 \\
\hline Рa-231 & 15 & \\
\hline $\mathrm{Pa}-233$ & 300 & \\
\hline $\mathrm{Pa}-234$ & $2.5 \mathrm{E}+03$ & \\
\hline \multicolumn{3}{|l|}{$\mathrm{Pa}-234 \mathrm{~m}$} \\
\hline $\mathrm{Pb}-202$ & $1.4 \mathrm{E}+02$ & \\
\hline $\mathrm{Pb}-205$ & $3.4 \mathrm{E}+03$ & \\
\hline $\mathrm{Pb}-209$ & $2.6 \mathrm{E}+04$ & \\
\hline $\mathrm{Pb}-210$ & $1.0 \mathrm{E}+00$ & \\
\hline $\mathrm{Pb}-211$ & $1.0 \mathrm{E}+04$ & \\
\hline
\end{tabular}


Table A-6 Groundwater Concentration Limits (pCi/L) (EPA 2002 and Eckerman 1988)

\begin{tabular}{|c|c|c|}
\hline Radionuclide & Primary Decay Mode & Secondary Decay Mode \\
\hline $\mathrm{Pb}-212$ & $1.2 \mathrm{E}+02$ & \\
\hline $\mathrm{Pb}-214$ & $8.8 \mathrm{E}+03$ & \\
\hline Pd-107 & $3.7 \mathrm{E}+04$ & \\
\hline Po-210 & 15 & \\
\hline Po-211 & 15 & \\
\hline Рo-212 & 15 & \\
\hline Po-213 & 15 & \\
\hline Рo-214 & 15 & \\
\hline Po-215 & 15 & \\
\hline Po-216 & 15 & \\
\hline Рo-218 & 15 & \\
\hline Pt-193 & 3000 & \\
\hline $\mathrm{Pu}-236$ & 15 & \\
\hline Pu-237 & $1.2 \mathrm{E}+04$ & \\
\hline Pu-238 & 15 & \\
\hline $\mathrm{Pu}-239$ & 15 & \\
\hline Pu-240 & 15 & \\
\hline $\mathrm{Pu}-241$ & 300 & \\
\hline Pu-242 & 15 & \\
\hline $\mathrm{Pu}-243$ & $1.6 \mathrm{E}+04$ & \\
\hline $\mathrm{Pu}-244$ & 15 & \\
\hline Pu-246 & $4.0 \mathrm{E}+02$ & \\
\hline Ra-222 & 15 & \\
\hline Ra-223 & 15 & \\
\hline $\mathrm{Ra}-224$ & 15 & \\
\hline Ra-225 & $1.4 \mathrm{E}+01$ & \\
\hline Ra-226 & 15 & \\
\hline Ra-228 & $3.8 \mathrm{E}+00$ & \\
\hline $\mathrm{Rb}-87$ & 300 & \\
\hline Re-186 & 300 & \\
\hline Re-186m & $1.4 \mathrm{E}+03$ & \\
\hline Re-187 & 9000 & \\
\hline Rn-218 & 15 & \\
\hline Rn-219 & 15 & \\
\hline $\mathrm{Rn}-220$ & 15 & \\
\hline Rn-222 & 15 & \\
\hline Ru-97 & 1000 & \\
\hline Sb-126 & $5.1 \mathrm{E}+02$ & \\
\hline $\mathrm{Sb}-126 \mathrm{~m}$ & $5.8 \mathrm{E}+04$ & \\
\hline Sc-44 & $3.8 \mathrm{E}+03$ & \\
\hline $\mathrm{Se}-79$ & $6.3 \mathrm{E}+02$ & \\
\hline Si-32 & $2.5 \mathrm{E}+03$ & \\
\hline
\end{tabular}


Table A-6 Groundwater Concentration Limits (pCi/L) (EPA 2002 and Eckerman 1988)

\begin{tabular}{|c|c|c|}
\hline Radionuclide & Primary Decay Mode & Secondary Decay Mode \\
\hline Sm-146 & 15 & \\
\hline Sm-147 & 15 & \\
\hline Sn-126 & $2.8 \mathrm{E}+02$ & \\
\hline Sr-90 & 8 & \\
\hline Та-180 & $1.5 \mathrm{E}+03$ & \\
\hline Тa-182 & 100 & \\
\hline Тc-97 & $3.2 \mathrm{E}+04$ & \\
\hline Tc-97m & $4.4 \mathrm{E}+03$ & \\
\hline Тc-98 & $1.1 \mathrm{E}+03$ & \\
\hline Tc-99 & 900 & \\
\hline Тe-123 & $1.3 \mathrm{E}+03$ & \\
\hline Th-226 & 15 & \\
\hline Th-227 & 15 & \\
\hline Th-228 & 15 & \\
\hline Th-229 & 15 & \\
\hline Th-230 & 15 & \\
\hline Th-231 & $4.1 \mathrm{E}+03$ & \\
\hline Th-232 & 15 & \\
\hline Th-234 & $4.0 \mathrm{E}+02$ & \\
\hline Ti-44 & $2.4 \mathrm{E}+02$ & \\
\hline Tl-202 & 300 & \\
\hline \multicolumn{3}{|l|}{ Tl-206 } \\
\hline \multicolumn{3}{|l|}{ Tl-207 } \\
\hline \multicolumn{3}{|l|}{ Tl-208 } \\
\hline \multicolumn{3}{|l|}{ Tl-209 } \\
\hline $\mathrm{U}-230$ & $8.2 \mathrm{E}+11(30 \mu \mathrm{g} / \mathrm{L})$ & \\
\hline U-232 & $6.7 \mathrm{E}+08(30 \mu \mathrm{g} / \mathrm{L})$ & \\
\hline U-233 & $2.9 \mathrm{E}+05(30 \mu \mathrm{g} / \mathrm{L})$ & \\
\hline U-234 & $1.9 \mathrm{E}+05(30 \mu \mathrm{g} / \mathrm{L})$ & \\
\hline U-235 & $6.5 \mathrm{E}+01(30 \mu \mathrm{g} / \mathrm{L})$ & \\
\hline U-236 & $1.9 \mathrm{E}+03(30 \mu \mathrm{g} / \mathrm{L})$ & \\
\hline U-237 & $2.4 \mathrm{E}+12(30 \mu \mathrm{g} / \mathrm{L})$ & \\
\hline U-238 & $1.0 \mathrm{E}+01(30 \mu \mathrm{g} / \mathrm{L})$ & \\
\hline U-240 & $2.8 \mathrm{E}+13(30 \mu \mathrm{g} / \mathrm{L})$ & \\
\hline$V-49$ & $8.9 \mathrm{E}+04$ & \\
\hline Y-90 & 60 & \\
\hline Zr-93 & 2000 & \\
\hline
\end{tabular}


Table A-7 Radionuclide Half Life and Branching Data (Tuli 2005)

\begin{tabular}{|c|c|c|c|c|c|c|c|}
\hline $\begin{array}{l}\text { Parent } \\
\text { Radionuclide }\end{array}$ & $\begin{array}{l}\text { Half Life } \\
\text { (years) }\end{array}$ & Daughter1 & Branch1 & Daughter2 & Branch2 & Daughter3 & Branch3 \\
\hline Ac- 225 & $2.7400 \mathrm{E}-02$ & Fr-221 & 1 & & & & \\
\hline Ac-227 & $2.1772 \mathrm{E}+01$ & Th-227 & 0.9862 & Fr-223 & 0.0138 & & \\
\hline Ac-228 & $7.0100 \mathrm{E}-04$ & Th-228 & 1 & & & & \\
\hline Ag-108 & $4.5000 \mathrm{E}-06$ & & & & & & \\
\hline $\mathrm{Ag}-108 \mathrm{~m}$ & $4.3800 \mathrm{E}+02$ & Ag-108 & 0.087 & & & & \\
\hline Al-26 & $7.1700 \mathrm{E}+05$ & & & & & & \\
\hline Am-237 & $1.3900 \mathrm{E}-04$ & $\mathrm{Pu}-237$ & 0.9998 & Np-233 & 0.0003 & & \\
\hline Am-241 & $4.3220 \mathrm{E}+02$ & $\mathrm{~Np}-237$ & 1 & & & & \\
\hline Am-243 & $7.3700 \mathrm{E}+03$ & $\mathrm{~Np}-239$ & 1 & & & & \\
\hline Am-245 & $2.3400 \mathrm{E}-04$ & $\mathrm{Cm}-245$ & 1 & & & & \\
\hline Am-246m & $4.7600 \mathrm{E}-05$ & $\mathrm{Cm}-246$ & 1 & & & & \\
\hline At-217 & $1.0200 \mathrm{E}-09$ & Bi-213 & 1 & & & & \\
\hline At-218 & $4.7600 \mathrm{E}-08$ & Bi-214 & 1 & & & & \\
\hline $\mathrm{Au}-194$ & $4.3400 \mathrm{E}-03$ & & & & & & \\
\hline Be-10 & $1.5100 \mathrm{E}+06$ & & & & & & \\
\hline Bi-210 & $1.3700 \mathrm{E}-02$ & Po-210 & 1 & & & & \\
\hline $\mathrm{Bi}-210 \mathrm{~m}$ & $3.0400 \mathrm{E}+06$ & Tl-206 & 1 & & & & \\
\hline Bi-211 & $4.0600 \mathrm{E}-06$ & Tl-207 & 0.9972 & Po-211 & 0.0028 & & \\
\hline Bi-212 & $1.1500 \mathrm{E}-04$ & Po-212 & 0.6406 & Tl-208 & 0.3594 & & \\
\hline Bi-213 & $8.6900 \mathrm{E}-05$ & Po-213 & 0.9791 & Tl-209 & 0.0209 & & \\
\hline Bi-214 & $3.7700 \mathrm{E}-05$ & Po-214 & 0.9998 & & & & \\
\hline Bk-247 & $1.3800 \mathrm{E}+03$ & Am-243 & 1 & & & & \\
\hline Bk-249 & $9.0400 \mathrm{E}-01$ & Cf- 249 & 1 & Am-245 & 0.000014 & & \\
\hline Bk-250 & $3.6800 \mathrm{E}-04$ & Cf-250 & 1 & & & & \\
\hline C-14 & $5.7300 \mathrm{E}+03$ & & & & & & \\
\hline $\mathrm{Ca}-41$ & $1.0200 \mathrm{E}+05$ & & & & & & \\
\hline Cd-113 & $7.7100 \mathrm{E}+15$ & & & & & & \\
\hline Cf-249 & $3.5200 \mathrm{E}+02$ & $\mathrm{Cm}-245$ & 1 & & & & \\
\hline Cf-250 & $1.3100 \mathrm{E}+01$ & $\mathrm{Cm}-246$ & 0.9992 & & & & \\
\hline Cf-251 & $8.9700 \mathrm{E}+02$ & $\mathrm{Cm}-247$ & 1 & & & & \\
\hline Cf- 252 & $2.6500 \mathrm{E}+00$ & $\mathrm{Cm}-248$ & 0.9691 & & & & \\
\hline Cl-36 & $3.0100 \mathrm{E}+05$ & & & & & & \\
\hline $\mathrm{Cm}-241$ & $8.9700 \mathrm{E}-02$ & Am-241 & 0.99 & Pu-237 & 0.01 & & \\
\hline $\mathrm{Cm}-242$ & $4.4700 \mathrm{E}-01$ & $\mathrm{Pu}-238$ & 1 & & & & \\
\hline $\mathrm{Cm}-244$ & $1.8100 \mathrm{E}+01$ & $\mathrm{Pu}-240$ & 1 & & & & \\
\hline $\mathrm{Cm}-245$ & $8.5000 \mathrm{E}+03$ & $\mathrm{Pu}-241$ & 1 & & & & \\
\hline $\mathrm{Cm}-246$ & $4.7600 \mathrm{E}+03$ & $\mathrm{Pu}-242$ & 0.9997 & & & & \\
\hline $\mathrm{Cm}-247$ & $1.5600 \mathrm{E}+07$ & $\mathrm{Pu}-243$ & 1 & & & & \\
\hline $\mathrm{Cm}-248$ & $3.4900 \mathrm{E}+05$ & Pu-244 & 0.9161 & & & & \\
\hline $\begin{array}{l}\mathrm{Cm}-250 \\
\mathrm{Co}-60\end{array}$ & $\begin{array}{l}8.3100 \mathrm{E}+03 \\
5.2600 \mathrm{E}+00\end{array}$ & Pu-246 & 0.18 & Bk-250 & 0.08 & & \\
\hline
\end{tabular}


Table A-7 Radionuclide Half Life and Branching Data (Tuli 2005)

\begin{tabular}{|c|c|c|c|c|c|c|c|}
\hline $\begin{array}{c}\text { Parent } \\
\text { Radionuclide }\end{array}$ & $\begin{array}{l}\text { Half Life } \\
\text { (years) }\end{array}$ & Daughter1 & Branch1 & Daughter2 & Branch2 & Daughter3 & Branch3 \\
\hline $\mathrm{Co}-60 \mathrm{~m}$ & $1.9900 \mathrm{E}-05$ & Co-60 & 0.9976 & & & & \\
\hline Cs-135 & $2.3000 \mathrm{E}+06$ & & & & & & \\
\hline Es-253 & $5.6100 \mathrm{E}-02$ & Bk-249 & 1 & & & & \\
\hline $\mathrm{Fe}-60$ & $1.5000 \mathrm{E}+06$ & Co-60m & 1 & & & & \\
\hline Fr-221 & $9.3200 \mathrm{E}-06$ & At-217 & 1 & & & & \\
\hline Fr-223 & 4.1900E-05 & $\mathrm{Ra}-223$ & 1 & & & & \\
\hline Ga-68 & $1.2900 \mathrm{E}-04$ & & & & & & \\
\hline Gd-152 & $1.0800 \mathrm{E}+14$ & & & & & & \\
\hline Ge-68 & $7.4200 \mathrm{E}-01$ & Ga-68 & 1 & & & & \\
\hline $\mathrm{H}-3$ & $1.2320 \mathrm{E}+01$ & & & & & & \\
\hline Hf-182 & $8.9100 \mathrm{E}+06$ & Та-182 & 1 & & & & \\
\hline Hg-194 & $4.4400 \mathrm{E}+02$ & $\mathrm{Au}-194$ & 1 & & & & \\
\hline Нo-166m & $1.2000 \mathrm{E}+03$ & & & & & & \\
\hline I-129 & $1.5700 \mathrm{E}+07$ & & & & & & \\
\hline In-115 & $4.4100 \mathrm{E}+14$ & & & & & & \\
\hline Ir-192 & $2.0200 \mathrm{E}-01$ & & & & & & \\
\hline Ir-192m & $2.4100 \mathrm{E}+02$ & Ir-192 & 1 & & & & \\
\hline K-40 & $1.2500 \mathrm{E}+09$ & & & & & & \\
\hline La-137 & $5.9900 \mathrm{E}+04$ & & & & & & \\
\hline La-138 & $1.0200 \mathrm{E}+11$ & & & & & & \\
\hline Lu-176 & $3.7700 \mathrm{E}+10$ & & & & & & \\
\hline $\mathrm{Mn}-53$ & $3.7400 \mathrm{E}+06$ & & & & & & \\
\hline Mo-93 & $4.0000 \mathrm{E}+03$ & $\mathrm{Nb}-93 \mathrm{~m}$ & 1 & & & & \\
\hline $\mathrm{Nb}-93 \mathrm{~m}$ & $1.6100 \mathrm{E}+01$ & & & & & & \\
\hline Nb-94 & $2.0300 \mathrm{E}+04$ & & & & & & \\
\hline Ni-59 & $7.6100 \mathrm{E}+04$ & & & & & & \\
\hline Np-233 & $6.8800 \mathrm{E}-05$ & U-233 & 1 & & & & \\
\hline Np-236a & $1.5400 \mathrm{E}+05$ & U-236 & 0.873 & $\mathrm{Pu}-236$ & 0.125 & & \\
\hline Np-237 & $2.1500 \mathrm{E}+06$ & $\mathrm{~Pa}-233$ & 1 & & & & \\
\hline Np-239 & $6.4700 \mathrm{E}-03$ & Pu-239 & 1 & & & & \\
\hline $\mathrm{Np}-240 \mathrm{~m}$ & $1.3700 \mathrm{E}-05$ & $\mathrm{Pu}-240$ & 1 & & & & \\
\hline P-32 & $3.9000 \mathrm{E}-02$ & & & & & & \\
\hline $\mathrm{Pa}-230$ & $4.7600 \mathrm{E}-02$ & Th-230 & 0.916 & U-230 & 0.084 & & \\
\hline Pa-231 & $3.2700 \mathrm{E}+04$ & Ac-227 & 1 & & & & \\
\hline $\mathrm{Pa}-233$ & $7.3900 \mathrm{E}-02$ & U-233 & 1 & & & & \\
\hline Pa-234 & $7.6400 \mathrm{E}-04$ & U-234 & 1 & & & & \\
\hline $\mathrm{Pa}-234 \mathrm{~m}$ & $2.2300 \mathrm{E}-06$ & U-234 & 0.9984 & $\mathrm{~Pa}-234$ & 0.0016 & & \\
\hline $\mathrm{Pb}-202$ & $5.2600 \mathrm{E}+04$ & Tl-202 & 1 & & & & \\
\hline $\mathrm{Pb}-205$ & $1.7300 \mathrm{E}+07$ & & & & & & \\
\hline $\mathrm{Pb}-209$ & $3.7100 \mathrm{E}-04$ & & & & & & \\
\hline $\mathrm{Pb}-210$ & $2.2200 \mathrm{E}+01$ & Bi-210 & 1 & & & & \\
\hline $\mathrm{Pb}-211$ & $6.8800 \mathrm{E}-05$ & Bi-211 & 1 & & & & \\
\hline
\end{tabular}


Rev. 0

Table A-7 Radionuclide Half Life and Branching Data (Tuli 2005)

\begin{tabular}{|c|c|c|c|c|c|c|c|}
\hline $\begin{array}{c}\text { Parent } \\
\text { Radionuclide }\end{array}$ & $\begin{array}{l}\text { Half Life } \\
\text { (years) }\end{array}$ & Daughter1 & Branch1 & Daughter2 & Branch2 & Daughter3 & Branch3 \\
\hline $\mathrm{Pb}-212$ & $1.2100 \mathrm{E}-03$ & $\mathrm{Bi}-212$ & 1 & & & & \\
\hline $\mathrm{Pb}-214$ & $5.1100 \mathrm{E}-05$ & Bi-214 & 1 & & & & \\
\hline Pd-107 & $6.5000 \mathrm{E}+06$ & & & & & & \\
\hline Po-210 & $3.8100 \mathrm{E}-01$ & & & & & & \\
\hline Po-211 & $1.6400 \mathrm{E}-08$ & & & & & & \\
\hline Po-212 & $9.4800 \mathrm{E}-15^{*}$ & & & & & & \\
\hline Po-213 & $1.1600 \mathrm{E}-13$ & $\mathrm{~Pb}-209$ & 1 & & & & \\
\hline Po-214 & $5.2000 \mathrm{E}-12$ & $\mathrm{~Pb}-210$ & 1 & & & & \\
\hline Po-215 & $5.6400 \mathrm{E}-11$ & $\mathrm{~Pb}-211$ & 1 & & & & \\
\hline Po-216 & 4.6000E-09 & $\mathrm{Pb}-212$ & 1 & & & & \\
\hline Po-218 & $5.9000 \mathrm{E}-06$ & $\mathrm{~Pb}-214$ & 0.9998 & At-218 & 0.0002 & & \\
\hline Pt-193 & $5.0100 \mathrm{E}+01$ & & & & & & \\
\hline Pu-236 & $2.8600 \mathrm{E}+00$ & U-232 & 1 & & & & \\
\hline $\mathrm{Pu}-237$ & $1.2400 \mathrm{E}-01$ & Np-237 & 1 & U-233 & 0.000042 & & \\
\hline Pu-238 & $8.7800 \mathrm{E}+01$ & U-234 & 1 & & & & \\
\hline $\mathrm{Pu}-239$ & $2.4100 \mathrm{E}+04$ & U-235 & 1 & & & & \\
\hline $\mathrm{Pu}-240$ & $6.5600 \mathrm{E}+03$ & U-236 & 1 & & & & \\
\hline $\mathrm{Pu}-241$ & $1.4300 \mathrm{E}+01$ & Am-241 & 1 & U-237 & 0.000025 & & \\
\hline Pu-242 & $3.7400 \mathrm{E}+05$ & U-238 & 1 & & & & \\
\hline Pu-243 & $5.6400 \mathrm{E}-04$ & Am-243 & 1 & & & & \\
\hline $\mathrm{Pu}-244$ & $7.9900 \mathrm{E}+07$ & U-240 & 0.9988 & & & & \\
\hline $\mathrm{Pu}-246$ & $2.9700 \mathrm{E}-02$ & Am-246m & 1 & & & & \\
\hline $\mathrm{Ra}-222$ & $1.2000 \mathrm{E}-06$ & Rn-218 & 1 & & & & \\
\hline Ra-223 & $3.1300 \mathrm{E}-02$ & Rn-219 & 1 & & & & \\
\hline Ra-224 & $9.9600 \mathrm{E}-03$ & Rn-220 & 1 & & & & \\
\hline Ra-225 & $4.0900 \mathrm{E}-02$ & Ac-225 & 1 & & & & \\
\hline Ra-226 & $1.6000 \mathrm{E}+03$ & Rn-222 & 1 & & & & \\
\hline $\mathrm{Ra}-228$ & $5.7400 \mathrm{E}+00$ & Ac-228 & 1 & & & & \\
\hline $\mathrm{Rb}-87$ & $4.9800 \mathrm{E}+10$ & & & & & & \\
\hline Re-186 & $1.0200 \mathrm{E}-02$ & & & & & & \\
\hline Re-186m & $2.0000 \mathrm{E}+05$ & $\operatorname{Re}-186$ & 1 & & & & \\
\hline Re-187 & $4.1200 \mathrm{E}+10$ & & & & & & \\
\hline Rn-218 & $1.1100 \mathrm{E}-09$ & Po-214 & 1 & & & & \\
\hline Rn-219 & $1.2600 \mathrm{E}-07$ & Po-215 & 1 & & & & \\
\hline $\mathrm{Rn}-220$ & $1.7600 \mathrm{E}-06$ & Po-216 & 1 & & & & \\
\hline $\mathrm{Rn}-222$ & $1.0500 \mathrm{E}-02$ & Po-218 & 1 & & & & \\
\hline Ru-97 & $7.6400 \mathrm{E}-03$ & Тc-97 & 1 & & & & \\
\hline Sb-126 & $3.3900 \mathrm{E}-02$ & & & & & & \\
\hline Sb-126m & $3.6500 \mathrm{E}-05$ & Sb-126 & 1 & & & & \\
\hline Sc-44 & $4.5300 \mathrm{E}-04$ & & & & & & \\
\hline $\mathrm{Se}-79$ & $2.9500 \mathrm{E}+05$ & & & & & & \\
\hline $\mathrm{Si}-32$ & $1.3200 \mathrm{E}+02$ & P-32 & 1 & & & & \\
\hline
\end{tabular}


Table A-7 Radionuclide Half Life and Branching Data (Tuli 2005)

\begin{tabular}{|c|c|c|c|c|c|c|c|}
\hline $\begin{array}{c}\text { Parent } \\
\text { Radionuclide }\end{array}$ & $\begin{array}{l}\text { Half Life } \\
\text { (years) }\end{array}$ & Daughter1 & Branch1 & Daughter2 & Branch2 & Daughter3 & Branch3 \\
\hline Sm-146 & $1.0300 \mathrm{E}+08$ & & & & & & \\
\hline Sm-147 & $1.0600 \mathrm{E}+11$ & & & & & & \\
\hline Sn-126 & $2.3000 \mathrm{E}+05$ & $\mathrm{Sb}-126 \mathrm{~m}$ & 1 & & & & \\
\hline Sr-90 & $2.8900 \mathrm{E}+01$ & Y-90 & 1 & & & & \\
\hline Та-180 & $1.2000 \mathrm{E}+15$ & & & & & & \\
\hline Тa-182 & $3.1400 \mathrm{E}-01$ & & & & & & \\
\hline Тc-97 & $4.2100 \mathrm{E}+06$ & & & & & & \\
\hline Tc-97m & $2.5100 \mathrm{E}-01$ & Tc-97 & 1 & & & & \\
\hline Тc-98 & $4.2000 \mathrm{E}+06$ & & & & & & \\
\hline Тc-99 & $2.1110 \mathrm{E}+05$ & & & & & & \\
\hline Те-123 & $9.2000 \mathrm{E}+16$ & & & & & & \\
\hline Th-226 & $5.8000 \mathrm{E}-05$ & Ra-222 & 1 & & & & \\
\hline Th-227 & $5.1100 \mathrm{E}-02$ & Ra-223 & 1 & & & & \\
\hline Th-228 & $1.9100 \mathrm{E}+00$ & $\mathrm{Ra}-224$ & 1 & & & & \\
\hline Th-229 & $7.3600 \mathrm{E}+03$ & Ra-225 & 1 & & & & \\
\hline Th-230 & $7.5500 \mathrm{E}+04$ & $\mathrm{Ra}-226$ & 1 & & & & \\
\hline Th-231 & $2.9100 \mathrm{E}-03$ & Pa-231 & 1 & & & & \\
\hline Th-232 & $1.4050 \mathrm{E}+10$ & Ra-228 & 1 & & & & \\
\hline Th-234 & $6.6000 \mathrm{E}-02$ & $\mathrm{~Pa}-234 \mathrm{~m}$ & 1 & & & & \\
\hline Ti-44 & $6.0000 \mathrm{E}+01$ & Sc-44 & 1 & & & & \\
\hline Tl-202 & $3.3600 \mathrm{E}-02$ & & & & & & \\
\hline Tl-206 & $7.9900 \mathrm{E}-06$ & & & & & & \\
\hline Tl-207 & $9.0700 \mathrm{E}-06$ & & & & & & \\
\hline Tl-208 & $5.8000 \mathrm{E}-06$ & & & & & & \\
\hline Tl-209 & $4.1200 \mathrm{E}-06$ & $\mathrm{~Pb}-209$ & 1 & & & & \\
\hline U-230 & $5.7100 \mathrm{E}-02$ & Th-226 & 1 & & & & \\
\hline U-232 & $6.8900 \mathrm{E}+01$ & Th-228 & 1 & & & & \\
\hline U-233 & $1.5920 \mathrm{E}+05$ & Th-229 & 1 & & & & \\
\hline U-234 & $2.4550 \mathrm{E}+05$ & Th-230 & 1 & & & & \\
\hline U-235 & $7.0400 \mathrm{E}+08$ & Th-231 & 1 & & & & \\
\hline U-236 & $2.3420 \mathrm{E}+07$ & Th-232 & 1 & & & & \\
\hline U-237 & $1.8500 \mathrm{E}-02$ & Np-237 & 1 & & & & \\
\hline U-238 & $4.4680 \mathrm{E}+09$ & Th-234 & 1 & & & & \\
\hline U-240 & $1.6100 \mathrm{E}-03$ & $\mathrm{~Np}-240 \mathrm{~m}$ & 1 & & & & \\
\hline V-49 & $9.0100 \mathrm{E}-01$ & & & & & & \\
\hline Y-90 & $7.3200 \mathrm{E}-03$ & & & & & & \\
\hline Zr-93 & $1.5300 \mathrm{E}+06$ & $\mathrm{Nb}-93 \mathrm{~m}$ & 1 & & & & \\
\hline
\end{tabular}

* Half life of Po-212 from Radionuclide Transformations: Energy and Intensity of Emissions. International Commission on Radiological Protection Report 38. Pergamon Press, Oxford. 1983. 
Rev. 0

Table A-8 Radionuclide Decay Modes (Tuli 2005)

\section{Decay Mode (percentage if} dual mode)

Non-

Nuclide

Ac- 225

Ac- 227

Ac- 228

Ag-108

Ag- $108 \mathrm{~m}$

Al-26

Am-237

Am-241

Am-243

Am-245

Am-246m

At-217

At-218

Au-194

Be-10

Bi-210

Bi-210m

Bi-211

Bi-212

Bi-213

Bi-214

Bk-247

Bk-249

Bk-250

C-14

Ca-41

Cd-113

Cf-249

Cf-250

Cf-251

Cf- $252^{1}$

Cl-36

Cm-241

Cm-242

Cm-244

Cm-245

Cm-246

Cm-247

Cm-248 ${ }^{1}$

\begin{tabular}{|c|c|}
\hline Alph & Alpha \\
\hline 99 & 1 \\
\hline$X$ & \\
\hline $\mathrm{X}$ & \\
\hline$X$ & \\
\hline$X$ & \\
\hline$X$ & \\
\hline & $\begin{array}{l}X \\
X\end{array}$ \\
\hline $\mathrm{X}$ & \\
\hline$X$ & \\
\hline & $\begin{array}{l}X \\
X\end{array}$ \\
\hline X & \\
\hline $\mathrm{X}$ & \\
\hline$X$ & \\
\hline & $\begin{array}{l}X \\
X\end{array}$ \\
\hline 64 & 36 \\
\hline 98 & 2 \\
\hline $\mathrm{X}$ & \\
\hline & $X$ \\
\hline $\begin{array}{l}\hat{x} \\
\mathrm{X}\end{array}$ & \\
\hline$X$ & \\
\hline X & \\
\hline$X$ & \\
\hline & X \\
\hline & X \\
\hline & X \\
\hline & 97 \\
\hline $\begin{array}{l}X \\
00\end{array}$ & \\
\hline 99 & $\begin{array}{l}1 \\
\mathrm{X}\end{array}$ \\
\hline & $\begin{array}{l}X \\
X\end{array}$ \\
\hline & $\mathrm{X}$ \\
\hline & $\begin{array}{l}X \\
X\end{array}$ \\
\hline & 92 \\
\hline
\end{tabular}


Table A-8 Radionuclide Decay Modes (Tuli 2005)

\begin{tabular}{|c|c|c|}
\hline & Decay $\mathrm{N}$ & $\begin{array}{l}\text { ercentage if } \\
\text { ode) }\end{array}$ \\
\hline Nuclide & $\begin{array}{l}\text { Non- } \\
\text { Alpha }\end{array}$ & Alpha \\
\hline $\mathrm{Cm}-250^{1}$ & 8 & 18 \\
\hline Co-60 & $\mathrm{X}$ & \\
\hline Co-60m & $\mathrm{X}$ & \\
\hline Cs-135 & $\mathrm{X}$ & \\
\hline Es-253 & & $X$ \\
\hline $\mathrm{Fe}-60$ & $X$ & \\
\hline Fr-221 & & $X$ \\
\hline Fr-223 & $\mathrm{X}$ & \\
\hline $\mathrm{Ga}-68$ & $\mathrm{X}$ & \\
\hline Gd-152 & & $X$ \\
\hline Ge-68 & $\mathrm{X}$ & \\
\hline $\mathrm{H}-3$ & $\mathrm{X}$ & \\
\hline Hf-182 & $\mathrm{X}$ & \\
\hline $\mathrm{Hg}-194$ & $\mathrm{X}$ & \\
\hline Но- $166 \mathrm{~m}$ & $\mathrm{X}$ & \\
\hline $\mathrm{I}-129$ & $\mathrm{X}$ & \\
\hline In-115 & $\mathrm{X}$ & \\
\hline Ir-192 & $\mathrm{X}$ & \\
\hline Ir-192m & $X$ & \\
\hline $\mathrm{K}-40$ & $\mathrm{X}$ & \\
\hline La-137 & $\mathrm{X}$ & \\
\hline La-138 & $\mathrm{X}$ & \\
\hline Lu-176 & $\mathrm{X}$ & \\
\hline Mn-53 & $\mathrm{X}$ & \\
\hline Mo-93 & $\mathrm{X}$ & \\
\hline $\mathrm{Nb}-93 \mathrm{~m}$ & $\mathrm{X}$ & \\
\hline $\mathrm{Nb}-94$ & $\mathrm{X}$ & \\
\hline $\mathrm{Ni}-59$ & $\mathrm{X}$ & \\
\hline Np-233 & $\mathrm{X}$ & \\
\hline Np-236a & $\mathrm{X}$ & \\
\hline Np-237 & & $X$ \\
\hline Np-239 & $\mathrm{X}$ & \\
\hline $\mathrm{Np}-240 \mathrm{~m}$ & $\mathrm{X}$ & \\
\hline P-32 & $\mathrm{X}$ & \\
\hline $\mathrm{Pa}-230$ & $X$ & \\
\hline Pa-231 & & $X$ \\
\hline $\mathrm{Pa}-233$ & $\mathrm{X}$ & \\
\hline $\mathrm{Pa}-234$ & $\mathrm{X}$ & \\
\hline $\mathrm{Pa}-234 \mathrm{~m}$ & $\mathrm{X}$ & \\
\hline $\mathrm{Pb}-202$ & $\mathrm{x}$ & \\
\hline
\end{tabular}


Table A-8 Radionuclide Decay Modes (Tuli 2005)

\begin{tabular}{|c|c|c|}
\hline & Decay $M$ & $\begin{array}{l}\text { ercenta } \\
\text { de) }\end{array}$ \\
\hline Nuclide & $\begin{array}{l}\text { Non- } \\
\text { Alpha }\end{array}$ & Alpha \\
\hline $\mathrm{Pb}-205$ & X & \\
\hline $\mathrm{Pb}-209$ & $\mathrm{X}$ & \\
\hline $\mathrm{Pb}-210$ & $\mathrm{X}$ & \\
\hline $\mathrm{Pb}-211$ & $\mathrm{X}$ & \\
\hline $\mathrm{Pb}-212$ & $X$ & \\
\hline $\mathrm{Pb}-214$ & $\mathrm{X}$ & \\
\hline Pd-107 & $X$ & \\
\hline Po-210 & & $\mathrm{X}$ \\
\hline Рo-211 & & $X$ \\
\hline Po- $212^{2}$ & & $\mathrm{X}$ \\
\hline Po-213 & & $X$ \\
\hline Po-214 & & $X$ \\
\hline Рo-215 & & $X$ \\
\hline Po-216 & & $X$ \\
\hline Po-218 & & $\mathrm{X}$ \\
\hline Pt-193 & $X$ & \\
\hline $\mathrm{Pu}-236$ & & $X$ \\
\hline $\mathrm{Pu}-237$ & $X$ & \\
\hline $\mathrm{Pu}-238$ & & $\mathrm{X}$ \\
\hline Pu-239 & & $X$ \\
\hline $\mathrm{Pu}-240$ & & $X$ \\
\hline $\mathrm{Pu}-241$ & $\mathrm{X}$ & \\
\hline $\mathrm{Pu}-242$ & & $X$ \\
\hline $\mathrm{Pu}-243$ & $X$ & \\
\hline $\mathrm{Pu}-244$ & & $X$ \\
\hline $\mathrm{Pu}-246$ & $X$ & \\
\hline Ra-222 & & $X$ \\
\hline $\mathrm{Ra}-223$ & & $X$ \\
\hline $\mathrm{Ra}-224$ & & $X$ \\
\hline $\mathrm{Ra}-225$ & $X$ & \\
\hline Ra-226 & & $X$ \\
\hline $\mathrm{Ra}-228$ & $\mathrm{X}$ & \\
\hline $\mathrm{Rb}-87$ & $X$ & \\
\hline Re-186 & $\mathrm{X}$ & \\
\hline Re-186m & $X$ & \\
\hline Re-187 & $\mathrm{X}$ & \\
\hline Rn-218 & & $X$ \\
\hline Rn-219 & & $X$ \\
\hline$R n-220$ & & $X$ \\
\hline $\mathrm{Rn}-222$ & & $X$ \\
\hline
\end{tabular}


Table A-8 Radionuclide Decay Modes (Tuli 2005)

$\begin{array}{lcc} & \text { Decay Mode (percentage if } \\ & \text { Non- } \\ \text { dual mode) }\end{array}$


Rev. 0

Table A-8 Radionuclide Decay Modes

(Tuli 2005)

\begin{tabular}{lcl} 
& \multicolumn{2}{c}{$\begin{array}{c}\text { Decay Mode (percentage if } \\
\text { dual mode) }\end{array}$} \\
Nuclide & Non- & \\
Alpha & Alpha \\
V-49 & $\mathrm{X}$ & \\
Y-90 & $\mathrm{X}$ \\
Zr-93 & $\mathrm{X}$
\end{tabular}

${ }^{1}$ The percentage of decays not accounted for in this table for Cf- $252, \mathrm{Cm}-248$ and $\mathrm{Cm}-250$ are due to spontaneous fission, which is not specifically accounted for in this PA.

${ }^{2}$ Decay mode for Po-212 from Radionuclide Transformations: Energy and Intensity of Emissions. International Commission on Radiological Protection Report 38. Pergamon Press, Oxford, 1983. 
Table A-9 Ingestion Effective Dose Conversion Factors (Eckerman et al. 1988)

\begin{tabular}{|c|c|c|c|c|}
\hline Radionuclide & $\mathrm{Sv} / \mathrm{Bq}$ & $\mathrm{mrem} / \mathrm{pCi}$ & $\mathrm{rem} / \mathrm{uCi}$ & $\begin{array}{c}\text { Gastrointestinal } \\
\text { Absorption } \\
\text { Factor (f1), if } \\
\text { more than one } \\
\text { is used from } \\
\text { Eckerman } \\
1988^{1}\end{array}$ \\
\hline Ac- 225 & $3.00 \mathrm{E}-08$ & $1.11 \mathrm{E}-04$ & $1.11 \mathrm{E}-01$ & \\
\hline Ac- 227 & $3.80 \mathrm{E}-06$ & $1.41 \mathrm{E}-02$ & $1.41 \mathrm{E}+01$ & \\
\hline Ac- 228 & $5.85 \mathrm{E}-10$ & $2.16 \mathrm{E}-06$ & $2.16 \mathrm{E}-03$ & \\
\hline Ag-108 & $0.00 \mathrm{E}+00$ & $0.00 \mathrm{E}+00$ & $0.00 \mathrm{E}+00$ & \\
\hline Ag-108m & $2.06 \mathrm{E}-09$ & $7.62 \mathrm{E}-06$ & $7.62 \mathrm{E}-03$ & \\
\hline $\mathrm{Al}-26$ & $3.94 \mathrm{E}-09$ & $1.46 \mathrm{E}-05$ & $1.46 \mathrm{E}-02$ & \\
\hline Am-237 & $1.78 \mathrm{E}-11$ & $6.59 \mathrm{E}-08$ & $6.59 \mathrm{E}-05$ & \\
\hline Am-241 & $9.84 \mathrm{E}-07$ & $3.64 \mathrm{E}-03$ & $3.64 \mathrm{E}+00$ & \\
\hline Am-243 & 9.79E-07 & $3.62 \mathrm{E}-03$ & $3.62 \mathrm{E}+00$ & \\
\hline Am-245 & $4.88 \mathrm{E}-11$ & $1.81 \mathrm{E}-07$ & $1.81 \mathrm{E}-04$ & \\
\hline Am-246m & $2.54 \mathrm{E}-11$ & $9.40 \mathrm{E}-08$ & $9.40 \mathrm{E}-05$ & \\
\hline At-217 & $0.00 \mathrm{E}+00$ & $0.00 \mathrm{E}+00$ & $0.00 \mathrm{E}+00$ & \\
\hline At-218 & $0.00 \mathrm{E}+00$ & $0.00 \mathrm{E}+00$ & $0.00 \mathrm{E}+00$ & \\
\hline Au-194 & $5.08 \mathrm{E}-10$ & $1.88 \mathrm{E}-06$ & $1.88 \mathrm{E}-03$ & \\
\hline Be-10 & $1.26 \mathrm{E}-09$ & $4.66 \mathrm{E}-06$ & $4.66 \mathrm{E}-03$ & \\
\hline $\mathrm{Bi}-210$ & $1.73 \mathrm{E}-09$ & $6.40 \mathrm{E}-06$ & $6.40 \mathrm{E}-03$ & \\
\hline $\mathrm{Bi}-210 \mathrm{~m}$ & $2.59 \mathrm{E}-08$ & $9.58 \mathrm{E}-05$ & $9.58 \mathrm{E}-02$ & \\
\hline $\mathrm{Bi}-211$ & $0.00 \mathrm{E}+00$ & $0.00 \mathrm{E}+00$ & $0.00 \mathrm{E}+00$ & \\
\hline $\mathrm{Bi}-212$ & $2.87 \mathrm{E}-10$ & $1.06 \mathrm{E}-06$ & $1.06 \mathrm{E}-03$ & \\
\hline $\mathrm{Bi}-213$ & $1.95 \mathrm{E}-10$ & $7.22 \mathrm{E}-07$ & 7.22E-04 & \\
\hline Bi-214 & $7.64 \mathrm{E}-11$ & $2.83 \mathrm{E}-07$ & $2.83 \mathrm{E}-04$ & \\
\hline Bk-247 & $1.27 \mathrm{E}-06$ & 4.70E-03 & $4.70 \mathrm{E}+00$ & \\
\hline Bk-249 & $3.24 \mathrm{E}-09$ & $1.20 \mathrm{E}-05$ & $1.20 \mathrm{E}-02$ & \\
\hline $\mathrm{Bk}-250$ & $1.57 \mathrm{E}-10$ & $5.81 \mathrm{E}-07$ & $5.81 \mathrm{E}-04$ & \\
\hline C-14 & $5.64 \mathrm{E}-10$ & $2.09 \mathrm{E}-06$ & $2.09 \mathrm{E}-03$ & \\
\hline $\mathrm{Ca}-41$ & $3.44 \mathrm{E}-10$ & $1.27 \mathrm{E}-06$ & $1.27 \mathrm{E}-03$ & \\
\hline Cd-113 & 4.70E-08 & $1.74 \mathrm{E}-04$ & $1.74 \mathrm{E}-01$ & \\
\hline Cf-249 & $1.28 \mathrm{E}-06$ & 4.74E-03 & $4.74 \mathrm{E}+00$ & \\
\hline $\mathrm{Cf}-250$ & $5.76 \mathrm{E}-07$ & $2.13 \mathrm{E}-03$ & $2.13 E+00$ & \\
\hline $\mathrm{Cf}-251$ & $1.31 \mathrm{E}-06$ & $4.85 \mathrm{E}-03$ & $4.85 \mathrm{E}+00$ & \\
\hline Cf-252 & $2.93 \mathrm{E}-07$ & $1.08 \mathrm{E}-03$ & $1.08 \mathrm{E}+00$ & \\
\hline $\mathrm{Cl}-36$ & $8.18 \mathrm{E}-10$ & $3.03 \mathrm{E}-06$ & $3.03 \mathrm{E}-03$ & \\
\hline $\mathrm{Cm}-241$ & $1.21 \mathrm{E}-09$ & $4.48 \mathrm{E}-06$ & $4.48 \mathrm{E}-03$ & \\
\hline $\mathrm{Cm}-242$ & $3.10 \mathrm{E}-08$ & $1.15 \mathrm{E}-04$ & $1.15 \mathrm{E}-01$ & \\
\hline $\mathrm{Cm}-244$ & $5.45 \mathrm{E}-07$ & $2.02 \mathrm{E}-03$ & $2.02 \mathrm{E}+00$ & \\
\hline
\end{tabular}


Table A-9 Ingestion Effective Dose Conversion Factors (Eckerman et al. 1988)

\begin{tabular}{|c|c|c|c|c|}
\hline Radionuclide & $\mathrm{Sv} / \mathrm{Bq}$ & $\mathrm{mrem} / \mathrm{pCi}$ & $\mathrm{rem} / \mathrm{uCi}$ & $1988^{1}$ \\
\hline $\mathrm{Cm}-245$ & $1.01 \mathrm{E}-06$ & $3.74 \mathrm{E}-03$ & $3.74 \mathrm{E}+00$ & \\
\hline $\mathrm{Cm}-246$ & $1.00 \mathrm{E}-06$ & $3.70 \mathrm{E}-03$ & $3.70 \mathrm{E}+00$ & \\
\hline Cm-247 & 9.24E-07 & $3.42 \mathrm{E}-03$ & $3.42 \mathrm{E}+00$ & \\
\hline $\mathrm{Cm}-248$ & $3.68 \mathrm{E}-06$ & $1.36 \mathrm{E}-02$ & $1.36 \mathrm{E}+01$ & \\
\hline $\mathrm{Cm}-250$ & $2.10 \mathrm{E}-05$ & $7.77 \mathrm{E}-02$ & $7.77 \mathrm{E}+01$ & \\
\hline Сo-60 & $7.28 \mathrm{E}-09$ & $2.69 \mathrm{E}-05$ & 2.69E-02 & $\mathrm{fl}=3 \mathrm{E}-1$ \\
\hline Сo-60 & $2.77 \mathrm{E}-09$ & $1.02 \mathrm{E}-05$ & $1.02 \mathrm{E}-02$ & $\mathrm{fl}=5 \mathrm{E}-2$ \\
\hline Co-60m & $9.82 \mathrm{E}-13$ & 3.63E-09 & 3.63E-06 & $\mathrm{fl}=3 \mathrm{E}-1$ \\
\hline Co-60m & $9.70 \mathrm{E}-13$ & $3.59 \mathrm{E}-09$ & $3.59 \mathrm{E}-06$ & $\mathrm{fl}=5 \mathrm{E}-2$ \\
\hline Cs-135 & $1.91 \mathrm{E}-09$ & 7.07E-06 & 7.07E-03 & \\
\hline Es-253 & $9.10 \mathrm{E}-09$ & $3.37 \mathrm{E}-05$ & $3.37 \mathrm{E}-02$ & \\
\hline $\mathrm{Fe}-60$ & $4.12 \mathrm{E}-08$ & $1.52 \mathrm{E}-04$ & $1.52 \mathrm{E}-01$ & \\
\hline Fr-221 & $0.00 \mathrm{E}+00$ & $0.00 \mathrm{E}+00$ & $0.00 \mathrm{E}+00$ & \\
\hline Fr-223 & 2.33E-09 & $8.62 \mathrm{E}-06$ & $8.62 \mathrm{E}-03$ & \\
\hline Ga-68 & $9.24 \mathrm{E}-11$ & $3.42 \mathrm{E}-07$ & $3.42 \mathrm{E}-04$ & \\
\hline Gd-152 & 4.34E-08 & $1.61 \mathrm{E}-04$ & $1.61 \mathrm{E}-01$ & \\
\hline Ge-68 & $2.89 \mathrm{E}-10$ & $1.07 \mathrm{E}-06$ & $1.07 \mathrm{E}-03$ & \\
\hline $\mathrm{H}-3$ & $1.73 \mathrm{E}-11$ & $6.40 \mathrm{E}-08$ & $6.40 \mathrm{E}-05$ & \\
\hline Hf-182 & 4.29E-09 & $1.59 \mathrm{E}-05$ & $1.59 \mathrm{E}-02$ & \\
\hline Hg-194 & $7.78 \mathrm{E}-08$ & $2.88 \mathrm{E}-04$ & $2.88 \mathrm{E}-01$ & $\mathrm{fl}=1.0$ \\
\hline Hg-194 & $1.66 \mathrm{E}-09$ & $6.14 \mathrm{E}-06$ & $6.14 \mathrm{E}-03$ & $\mathrm{fl}=2 \mathrm{E}-2$ \\
\hline Ho-166m & $2.18 \mathrm{E}-09$ & 8.07E-06 & 8.07E-03 & \\
\hline I-129 & $7.46 \mathrm{E}-08$ & $2.76 \mathrm{E}-04$ & $2.76 \mathrm{E}-01$ & \\
\hline In-115 & $4.26 \mathrm{E}-08$ & $1.58 \mathrm{E}-04$ & $1.58 \mathrm{E}-01$ & \\
\hline Ir-192 & $1.55 \mathrm{E}-09$ & $5.74 \mathrm{E}-06$ & $5.74 \mathrm{E}-03$ & \\
\hline Ir-192m & $4.23 \mathrm{E}-10$ & $1.57 \mathrm{E}-06$ & $1.57 \mathrm{E}-03$ & \\
\hline $\mathrm{K}-40$ & $5.02 \mathrm{E}-09$ & $1.86 \mathrm{E}-05$ & $1.86 \mathrm{E}-02$ & \\
\hline La-137 & $1.23 \mathrm{E}-10$ & $4.55 \mathrm{E}-07$ & $4.55 \mathrm{E}-04$ & \\
\hline La-138 & $1.59 \mathrm{E}-09$ & $5.88 \mathrm{E}-06$ & $5.88 \mathrm{E}-03$ & \\
\hline Lu-176 & $1.98 \mathrm{E}-09$ & 7.33E-06 & $7.33 \mathrm{E}-03$ & \\
\hline $\mathrm{Mn}-53$ & $2.92 \mathrm{E}-11$ & $1.08 \mathrm{E}-07$ & $1.08 \mathrm{E}-04$ & \\
\hline Mo-93 & 3.64E-10 & $1.35 \mathrm{E}-06$ & $1.35 \mathrm{E}-03$ & $\mathrm{fl}=8 \mathrm{E}-1$ \\
\hline Nb-93m & $1.41 \mathrm{E}-10$ & $5.22 \mathrm{E}-07$ & $5.22 \mathrm{E}-04$ & \\
\hline Nb-94 & $1.93 \mathrm{E}-09$ & 7.14E-06 & 7.14E-03 & \\
\hline $\mathrm{Ni}-59$ & $5.67 \mathrm{E}-11$ & $2.10 \mathrm{E}-07$ & $2.10 \mathrm{E}-04$ & \\
\hline $\mathrm{Np}-233$ & $1.99 \mathrm{E}-12$ & $7.36 \mathrm{E}-09$ & $7.36 \mathrm{E}-06$ & \\
\hline
\end{tabular}


Table A-9 Ingestion Effective Dose Conversion Factors (Eckerman et al. 1988)

\begin{tabular}{|c|c|c|c|c|}
\hline Radionuclide & $\mathrm{Sv} / \mathrm{Bq}$ & $\mathrm{mrem} / \mathrm{pCi}$ & $\mathrm{rem} / \mathrm{uCi}$ & $1988^{1}$ \\
\hline Np-236a & $2.34 \mathrm{E}-07$ & $8.66 \mathrm{E}-04$ & $8.66 \mathrm{E}-01$ & \\
\hline Np-237 & $1.20 \mathrm{E}-06$ & 4.44E-03 & $4.44 \mathrm{E}+00$ & \\
\hline Np-239 & $8.82 \mathrm{E}-10$ & $3.26 \mathrm{E}-06$ & $3.26 \mathrm{E}-03$ & \\
\hline $\mathrm{Np}-240 \mathrm{~m}$ & $0.00 \mathrm{E}+00$ & $0.00 \mathrm{E}+00$ & $0.00 \mathrm{E}+00$ & \\
\hline P-32 & 2.37E-09 & $8.77 \mathrm{E}-06$ & $8.77 \mathrm{E}-03$ & \\
\hline $\mathrm{Pa}-230$ & $1.68 \mathrm{E}-09$ & $6.22 \mathrm{E}-06$ & $6.22 \mathrm{E}-03$ & \\
\hline $\mathrm{Pa}-231$ & $2.86 \mathrm{E}-06$ & $1.06 \mathrm{E}-02$ & $1.06 \mathrm{E}+01$ & \\
\hline $\mathrm{Pa}-233$ & $9.81 \mathrm{E}-10$ & $3.63 \mathrm{E}-06$ & $3.63 \mathrm{E}-03$ & \\
\hline $\mathrm{Pa}-234$ & $5.84 \mathrm{E}-10$ & 2.16E-06 & $2.16 \mathrm{E}-03$ & \\
\hline $\mathrm{Pa}-234 \mathrm{~m}$ & $0.00 \mathrm{E}+00$ & $0.00 \mathrm{E}+00$ & $0.00 \mathrm{E}+00$ & \\
\hline $\mathrm{Pb}-202$ & $1.05 \mathrm{E}-08$ & $3.89 \mathrm{E}-05$ & $3.89 \mathrm{E}-02$ & \\
\hline $\mathrm{Pb}-205$ & $4.41 \mathrm{E}-10$ & $1.63 \mathrm{E}-06$ & $1.63 \mathrm{E}-03$ & \\
\hline $\mathrm{Pb}-209$ & $5.75 \mathrm{E}-11$ & 2.13E-07 & $2.13 \mathrm{E}-04$ & \\
\hline $\mathrm{Pb}-210$ & $1.45 \mathrm{E}-06$ & $5.37 \mathrm{E}-03$ & $5.37 \mathrm{E}+00$ & \\
\hline $\mathrm{Pb}-211$ & $1.42 \mathrm{E}-10$ & $5.25 \mathrm{E}-07$ & $5.25 \mathrm{E}-04$ & \\
\hline $\mathrm{Pb}-212$ & $1.23 \mathrm{E}-08$ & $4.55 \mathrm{E}-05$ & $4.55 \mathrm{E}-02$ & \\
\hline $\mathrm{Pb}-214$ & $1.69 \mathrm{E}-10$ & $6.25 \mathrm{E}-07$ & $6.25 \mathrm{E}-04$ & \\
\hline Pd-107 & $4.04 \mathrm{E}-11$ & $1.49 \mathrm{E}-07$ & $1.49 \mathrm{E}-04$ & \\
\hline Po-210 & $5.14 \mathrm{E}-07$ & $1.90 \mathrm{E}-03$ & $1.90 \mathrm{E}+00$ & \\
\hline Po-211 & $0.00 \mathrm{E}+00$ & $0.00 \mathrm{E}+00$ & $0.00 \mathrm{E}+00$ & \\
\hline Po-212 & $0.00 \mathrm{E}+00$ & $0.00 \mathrm{E}+00$ & $0.00 \mathrm{E}+00$ & \\
\hline Po-213 & $0.00 \mathrm{E}+00$ & $0.00 \mathrm{E}+00$ & $0.00 \mathrm{E}+00$ & \\
\hline Po-214 & $0.00 \mathrm{E}+00$ & $0.00 \mathrm{E}+00$ & $0.00 \mathrm{E}+00$ & \\
\hline Po-215 & $0.00 \mathrm{E}+00$ & $0.00 \mathrm{E}+00$ & $0.00 \mathrm{E}+00$ & \\
\hline Po-216 & $0.00 \mathrm{E}+00$ & $0.00 \mathrm{E}+00$ & $0.00 \mathrm{E}+00$ & \\
\hline Рo-218 & $0.00 \mathrm{E}+00$ & $0.00 \mathrm{E}+00$ & $0.00 \mathrm{E}+00$ & \\
\hline Pt-193 & $3.21 \mathrm{E}-11$ & $1.19 \mathrm{E}-07$ & $1.19 \mathrm{E}-04$ & \\
\hline Pu-236 & $3.15 \mathrm{E}-07$ & $1.17 \mathrm{E}-03$ & $1.17 \mathrm{E}+00$ & $\mathrm{fl}=1 \mathrm{E}-3$ \\
\hline $\mathrm{Pu}-237$ & $1.20 \mathrm{E}-10$ & 4.44E-07 & 4.44E-04 & $\mathrm{f} 1=1 \mathrm{E}-3$ \\
\hline $\mathrm{Pu}-238$ & $8.65 \mathrm{E}-07$ & $3.20 \mathrm{E}-03$ & $3.20 \mathrm{E}+00$ & $\mathrm{fl}=1 \mathrm{E}-3$ \\
\hline Pu-239 & $9.56 \mathrm{E}-07$ & $3.54 \mathrm{E}-03$ & $3.54 \mathrm{E}+00$ & $\mathrm{fl}=1 \mathrm{E}-3$ \\
\hline Pu-240 & $9.56 \mathrm{E}-07$ & $3.54 \mathrm{E}-03$ & $3.54 \mathrm{E}+00$ & $f 1=1 E-3$ \\
\hline Pu-241 & $1.85 \mathrm{E}-08$ & $6.85 \mathrm{E}-05$ & $6.85 \mathrm{E}-02$ & $\mathrm{fl}=1 \mathrm{E}-3$ \\
\hline $\mathrm{Pu}-242$ & $9.08 \mathrm{E}-07$ & $3.36 \mathrm{E}-03$ & $3.36 \mathrm{E}+00$ & $\mathrm{f} 1=1 \mathrm{E}-3$ \\
\hline $\mathrm{Pu}-243$ & $9.02 \mathrm{E}-11$ & $3.34 \mathrm{E}-07$ & $3.34 \mathrm{E}-04$ & $\mathrm{fl}=1 \mathrm{E}-3$ \\
\hline Pu-244 & $8.97 \mathrm{E}-07$ & 3.32E-03 & $3.32 \mathrm{E}+00$ & $\mathrm{f} 1=1 \mathrm{E}-3$ \\
\hline
\end{tabular}


Table A-9 Ingestion Effective Dose Conversion Factors (Eckerman et al. 1988)

\begin{tabular}{|c|c|c|c|c|}
\hline Radionuclide & $\mathrm{Sv} / \mathrm{Bq}$ & mrem/pCi & $\mathrm{rem} / \mathrm{uCi}$ & $\begin{array}{c}\text { Gastrointestinal } \\
\text { Absorption } \\
\text { Factor (f1), if } \\
\text { more than one } \\
\text { is used from } \\
\text { Eckerman } \\
1988^{1}\end{array}$ \\
\hline $\mathrm{Pu}-246$ & $3.66 \mathrm{E}-09$ & $1.35 \mathrm{E}-05$ & $1.35 \mathrm{E}-02$ & \\
\hline $\mathrm{Ra}-222$ & $0.00 \mathrm{E}+00$ & $0.00 \mathrm{E}+00$ & $0.00 \mathrm{E}+00$ & \\
\hline $\mathrm{Ra}-223$ & $1.78 \mathrm{E}-07$ & $6.59 \mathrm{E}-04$ & $6.59 \mathrm{E}-01$ & \\
\hline $\mathrm{Ra}-224$ & 9.89E-08 & $3.66 \mathrm{E}-04$ & $3.66 \mathrm{E}-01$ & \\
\hline $\mathrm{Ra}-225$ & $1.04 \mathrm{E}-07$ & $3.85 \mathrm{E}-04$ & $3.85 \mathrm{E}-01$ & \\
\hline Ra-226 & $3.58 \mathrm{E}-07$ & $1.32 \mathrm{E}-03$ & $1.32 \mathrm{E}+00$ & \\
\hline $\mathrm{Ra}-228$ & $3.88 \mathrm{E}-07$ & $1.44 \mathrm{E}-03$ & $1.44 \mathrm{E}+00$ & \\
\hline $\mathrm{Rb}-87$ & $1.33 \mathrm{E}-09$ & 4.92E-06 & $4.92 \mathrm{E}-03$ & \\
\hline Re-186 & $7.95 \mathrm{E}-10$ & $2.94 \mathrm{E}-06$ & $2.94 \mathrm{E}-03$ & \\
\hline Re-186m & $1.08 \mathrm{E}-09$ & 4.00E-06 & $4.00 \mathrm{E}-03$ & \\
\hline $\operatorname{Re}-187$ & $2.57 \mathrm{E}-12$ & $9.51 \mathrm{E}-09$ & $9.51 \mathrm{E}-06$ & \\
\hline $\mathrm{Rn}-218$ & $0.00 \mathrm{E}+00$ & $0.00 \mathrm{E}+00$ & $0.00 \mathrm{E}+00$ & \\
\hline$R n-219$ & $0.00 \mathrm{E}+00$ & $0.00 \mathrm{E}+00$ & $0.00 \mathrm{E}+00$ & \\
\hline$R n-220$ & $0.00 \mathrm{E}+00$ & $0.00 \mathrm{E}+00$ & $0.00 \mathrm{E}+00$ & \\
\hline $\mathrm{Rn}-222$ & $0.00 \mathrm{E}+00$ & $0.00 \mathrm{E}+00$ & $0.00 \mathrm{E}+00$ & \\
\hline Ru-97 & $1.88 \mathrm{E}-10$ & $6.96 \mathrm{E}-07$ & $6.96 \mathrm{E}-04$ & \\
\hline $\mathrm{Sb}-126$ & 2.76E-09 & $1.02 \mathrm{E}-05$ & $1.02 \mathrm{E}-02$ & $\mathrm{fl}=1 \mathrm{E}-1$ \\
\hline $\mathrm{Sb}-126$ & 2.89E-09 & $1.07 \mathrm{E}-05$ & $1.07 \mathrm{E}-02$ & $\mathrm{fl}=1 \mathrm{E}-2$ \\
\hline $\mathrm{Sb}-126 \mathrm{~m}$ & $2.53 \mathrm{E}-11$ & $9.36 \mathrm{E}-08$ & $9.36 \mathrm{E}-05$ & $\mathrm{fl}=1 \mathrm{E}-1$ \\
\hline $\mathrm{Sb}-126 \mathrm{~m}$ & $2.54 \mathrm{E}-11$ & $9.40 \mathrm{E}-08$ & $9.40 \mathrm{E}-05$ & $\mathrm{fl}=1 \mathrm{E}-2$ \\
\hline Sc-44 & $3.87 \mathrm{E}-10$ & $1.43 \mathrm{E}-06$ & $1.43 \mathrm{E}-03$ & \\
\hline Se-79 & $2.35 \mathrm{E}-09$ & 8.70E-06 & $8.70 \mathrm{E}-03$ & $\mathrm{fl}=8 \mathrm{E}-1$ \\
\hline $\mathrm{Si}-32$ & $5.90 \mathrm{E}-10$ & $2.18 \mathrm{E}-06$ & $2.18 \mathrm{E}-03$ & \\
\hline Sm-146 & $5.51 \mathrm{E}-08$ & $2.04 \mathrm{E}-04$ & $2.04 \mathrm{E}-01$ & \\
\hline $\mathrm{Sm}-147$ & $5.01 \mathrm{E}-08$ & $1.85 \mathrm{E}-04$ & $1.85 \mathrm{E}-01$ & \\
\hline Sn-126 & 5.27E-09 & $1.95 \mathrm{E}-05$ & $1.95 \mathrm{E}-02$ & \\
\hline Sr-90 & $3.85 \mathrm{E}-08$ & $1.42 \mathrm{E}-04$ & $1.42 \mathrm{E}-01$ & $\mathrm{fl}=3 \mathrm{E}-1$ \\
\hline Та-180 & $9.82 \mathrm{E}-10$ & $3.63 \mathrm{E}-06$ & $3.63 \mathrm{E}-03$ & \\
\hline Тa-182 & $1.76 \mathrm{E}-09$ & $6.51 \mathrm{E}-06$ & $6.51 \mathrm{E}-03$ & \\
\hline Tc-97 & $4.63 \mathrm{E}-11$ & $1.71 \mathrm{E}-07$ & $1.71 \mathrm{E}-04$ & \\
\hline Tc-97m & $3.36 \mathrm{E}-10$ & $1.24 \mathrm{E}-06$ & $1.24 \mathrm{E}-03$ & \\
\hline Tc-98 & $1.32 \mathrm{E}-09$ & $4.88 \mathrm{E}-06$ & $4.88 \mathrm{E}-03$ & \\
\hline Тc-99 & $3.95 \mathrm{E}-10$ & $1.46 \mathrm{E}-06$ & $1.46 \mathrm{E}-03$ & \\
\hline Te-123 & 1.13E-09 & 4.18E-06 & 4.18E-03 & \\
\hline Th-226 & $2.50 \mathrm{E}-10$ & $9.25 \mathrm{E}-07$ & $9.25 \mathrm{E}-04$ & \\
\hline Th-227 & $1.03 \mathrm{E}-08$ & $3.81 \mathrm{E}-05$ & $3.81 \mathrm{E}-02$ & \\
\hline
\end{tabular}


Table A-9 Ingestion Effective Dose Conversion Factors (Eckerman et al. 1988)

\begin{tabular}{|c|c|c|c|c|}
\hline Radionuclide & $\mathrm{Sv} / \mathrm{Bq}$ & $\mathrm{mrem} / \mathrm{pCi}$ & $\mathrm{rem} / \mathrm{uCi}$ & $\begin{array}{l}\text { Gastrointestinal } \\
\text { Absorption } \\
\text { Factor (f1), if } \\
\text { more than one } \\
\text { is used from } \\
\text { Eckerman } \\
1988^{1}\end{array}$ \\
\hline Th-228 & $1.07 \mathrm{E}-07$ & $3.96 \mathrm{E}-04$ & $3.96 \mathrm{E}-01$ & \\
\hline Th-229 & $9.54 \mathrm{E}-07$ & $3.53 \mathrm{E}-03$ & $3.53 \mathrm{E}+00$ & \\
\hline Th-230 & $1.48 \mathrm{E}-07$ & $5.48 \mathrm{E}-04$ & $5.48 \mathrm{E}-01$ & \\
\hline Th-231 & $3.65 \mathrm{E}-10$ & $1.35 \mathrm{E}-06$ & $1.35 \mathrm{E}-03$ & \\
\hline Th-232 & $7.38 \mathrm{E}-07$ & $2.73 \mathrm{E}-03$ & $2.73 \mathrm{E}+00$ & \\
\hline Th-234 & 3.69E-09 & $1.37 \mathrm{E}-05$ & $1.37 \mathrm{E}-02$ & \\
\hline Ti-44 & $6.25 \mathrm{E}-09$ & $2.31 \mathrm{E}-05$ & $2.31 \mathrm{E}-02$ & \\
\hline Tl-202 & $3.98 \mathrm{E}-10$ & $1.47 \mathrm{E}-06$ & $1.47 \mathrm{E}-03$ & \\
\hline Tl-206 & $0.00 \mathrm{E}+00$ & $0.00 \mathrm{E}+00$ & $0.00 \mathrm{E}+00$ & \\
\hline Tl-207 & $0.00 \mathrm{E}+00$ & $0.00 \mathrm{E}+00$ & $0.00 \mathrm{E}+00$ & \\
\hline Tl-208 & $0.00 \mathrm{E}+00$ & $0.00 \mathrm{E}+00$ & $0.00 \mathrm{E}+00$ & \\
\hline T1-209 & $0.00 \mathrm{E}+00$ & $0.00 \mathrm{E}+00$ & $0.00 \mathrm{E}+00$ & \\
\hline U-230 & $2.44 \mathrm{E}-07$ & $9.03 \mathrm{E}-04$ & 9.03E-01 & $\mathrm{fl}=5 \mathrm{E}-2$ \\
\hline U-232 & $3.54 \mathrm{E}-07$ & $1.31 \mathrm{E}-03$ & $1.31 \mathrm{E}+00$ & $\mathrm{fl}=5 \mathrm{E}-2$ \\
\hline U-233 & $7.81 \mathrm{E}-08$ & $2.89 \mathrm{E}-04$ & $2.89 \mathrm{E}-01$ & $\mathrm{fl}=5 \mathrm{E}-2$ \\
\hline U-234 & $7.66 \mathrm{E}-08$ & $2.83 \mathrm{E}-04$ & $2.83 \mathrm{E}-01$ & $\mathrm{fl}=5 \mathrm{E}-2$ \\
\hline U-235 & 7.19E-08 & $2.66 \mathrm{E}-04$ & $2.66 \mathrm{E}-01$ & $\mathrm{fl}=5 \mathrm{E}-2$ \\
\hline U-236 & $7.26 \mathrm{E}-08$ & $2.69 \mathrm{E}-04$ & $2.69 \mathrm{E}-01$ & $\mathrm{fl}=5 \mathrm{E}-2$ \\
\hline U-237 & $8.48 \mathrm{E}-10$ & $3.14 \mathrm{E}-06$ & $3.14 \mathrm{E}-03$ & $\mathrm{fl}=5 \mathrm{E}-2$ \\
\hline U-238 & $6.88 \mathrm{E}-08$ & $2.55 \mathrm{E}-04$ & $2.55 \mathrm{E}-01$ & $\mathrm{fl}=5 \mathrm{E}-2$ \\
\hline U-240 & $1.16 \mathrm{E}-09$ & 4.29E-06 & $4.29 \mathrm{E}-03$ & \\
\hline$V-49$ & $1.66 \mathrm{E}-11$ & $6.14 \mathrm{E}-08$ & $6.14 \mathrm{E}-05$ & \\
\hline Y-90 & $2.91 \mathrm{E}-09$ & $1.08 \mathrm{E}-05$ & $1.08 \mathrm{E}-02$ & \\
\hline Zr-93 & $4.48 \mathrm{E}-10$ & $1.66 \mathrm{E}-06$ & $1.66 \mathrm{E}-03$ & \\
\hline
\end{tabular}

${ }^{1}$ In a few cases (Co-60, Co-60m, Hg-194, Sb-126 and Sb-126m), higher f1 values were used for the intruder than for the all pathways analyses. 
Table A-10 Radionuclide Decay Constants (1/day) (Tuli 2005)

\begin{tabular}{|c|c|c|c|}
\hline Radionuclide & Decay Constant & Radionuclide & Decay Constant \\
\hline Ac- 225 & $6.93 \mathrm{E}-02$ & Es-253 & $3.38 \mathrm{E}-02$ \\
\hline Ac- 227 & $8.72 \mathrm{E}-05$ & $\mathrm{Fe}-60$ & $1.27 \mathrm{E}-09$ \\
\hline Ac- 228 & $2.71 \mathrm{E}+00$ & Fr-221 & $2.04 \mathrm{E}+02$ \\
\hline Ag-108 & $4.22 \mathrm{E}+02$ & Fr-223 & $4.53 \mathrm{E}+01$ \\
\hline Ag-108m & $4.33 \mathrm{E}-06$ & Ga-68 & $1.47 \mathrm{E}+01$ \\
\hline Al-26 & $2.65 \mathrm{E}-09$ & Gd-152 & $1.76 \mathrm{E}-17$ \\
\hline Am-237 & $1.37 \mathrm{E}+01$ & Ge-68 & $2.56 \mathrm{E}-03$ \\
\hline Am-241 & $4.39 \mathrm{E}-06$ & $\mathrm{H}-3$ & $1.54 \mathrm{E}-04$ \\
\hline Am-243 & $2.57 \mathrm{E}-07$ & Hf-182 & $2.13 \mathrm{E}-10$ \\
\hline Am-245 & $8.11 \mathrm{E}+00$ & $\mathrm{Hg}-194$ & 4.27E-06 \\
\hline Am-246m & $3.99 \mathrm{E}+01$ & Ho- $166 \mathrm{~m}$ & $1.58 \mathrm{E}-06$ \\
\hline At-217 & $1.86 \mathrm{E}+06$ & $\mathrm{I}-129$ & $1.21 \mathrm{E}-10$ \\
\hline At-218 & $3.99 \mathrm{E}+04$ & In-115 & $4.30 \mathrm{E}-18$ \\
\hline Au-194 & 4.37E-01 & Ir-192 & 9.39E-03 \\
\hline $\mathrm{Be}-10$ & $1.26 \mathrm{E}-09$ & Ir- $192 \mathrm{~m}$ & $7.87 \mathrm{E}-06$ \\
\hline $\mathrm{Bi}-210$ & $1.39 \mathrm{E}-01$ & $\mathrm{~K}-40$ & $1.52 \mathrm{E}-12$ \\
\hline $\mathrm{Bi}-210 \mathrm{~m}$ & $6.24 \mathrm{E}-10$ & La-137 & $3.17 \mathrm{E}-08$ \\
\hline $\mathrm{Bi}-211$ & $4.67 \mathrm{E}+02$ & La-138 & $1.86 \mathrm{E}-14$ \\
\hline $\mathrm{Bi}-212$ & $1.65 \mathrm{E}+01$ & Lu-176 & $5.03 \mathrm{E}-14$ \\
\hline $\mathrm{Bi}-213$ & $2.18 \mathrm{E}+01$ & $M n-53$ & $5.07 \mathrm{E}-10$ \\
\hline $\mathrm{Bi}-214$ & $5.03 \mathrm{E}+01$ & Mo-93 & 4.74E-07 \\
\hline Bk-247 & $1.38 \mathrm{E}-06$ & $\mathrm{Nb}-93 \mathrm{~m}$ & $1.18 \mathrm{E}-04$ \\
\hline Bk-249 & $2.10 \mathrm{E}-03$ & $\mathrm{Nb}-94$ & $9.35 \mathrm{E}-08$ \\
\hline $\mathrm{Bk}-250$ & $5.16 \mathrm{E}+00$ & Ni-59 & $2.49 \mathrm{E}-08$ \\
\hline C-14 & $3.31 \mathrm{E}-07$ & $\mathrm{~Np}-233$ & $2.76 \mathrm{E}+01$ \\
\hline $\mathrm{Ca}-41$ & $1.86 \mathrm{E}-08$ & $\mathrm{~Np}-236 \mathrm{a}$ & $1.23 \mathrm{E}-08$ \\
\hline Cd-113 & $2.46 \mathrm{E}-19$ & $\mathrm{~Np}-237$ & $8.83 \mathrm{E}-10$ \\
\hline Cf-249 & $5.39 \mathrm{E}-06$ & $\mathrm{~Np}-239$ & $2.93 \mathrm{E}-01$ \\
\hline Cf-250 & $1.45 \mathrm{E}-04$ & $\mathrm{~Np}-240 \mathrm{~m}$ & $1.39 \mathrm{E}+02$ \\
\hline Cf-251 & $2.12 \mathrm{E}-06$ & P-32 & $4.87 \mathrm{E}-02$ \\
\hline Cf-252 & $7.16 \mathrm{E}-04$ & $\mathrm{~Pa}-230$ & $3.99 \mathrm{E}-02$ \\
\hline $\mathrm{Cl}-36$ & $6.30 \mathrm{E}-09$ & $\mathrm{~Pa}-231$ & $5.80 \mathrm{E}-08$ \\
\hline $\mathrm{Cm}-241$ & $2.12 \mathrm{E}-02$ & $\mathrm{~Pa}-233$ & $2.57 \mathrm{E}-02$ \\
\hline $\mathrm{Cm}-242$ & $4.25 \mathrm{E}-03$ & $\mathrm{~Pa}-234$ & $2.48 \mathrm{E}+00$ \\
\hline $\mathrm{Cm}-244$ & $1.05 \mathrm{E}-04$ & $\mathrm{~Pa}-234 \mathrm{~m}$ & $8.51 \mathrm{E}+02$ \\
\hline $\mathrm{Cm}-245$ & $2.23 \mathrm{E}-07$ & $\mathrm{~Pb}-202$ & $3.61 \mathrm{E}-08$ \\
\hline $\mathrm{Cm}-246$ & $3.99 \mathrm{E}-07$ & $\mathrm{~Pb}-205$ & $1.10 \mathrm{E}-10$ \\
\hline $\mathrm{Cm}-247$ & $1.22 \mathrm{E}-10$ & $\mathrm{~Pb}-209$ & $5.12 \mathrm{E}+00$ \\
\hline $\mathrm{Cm}-248$ & $5.44 \mathrm{E}-09$ & $\mathrm{~Pb}-210$ & $8.55 \mathrm{E}-05$ \\
\hline $\mathrm{Cm}-250$ & $2.28 \mathrm{E}-07$ & $\mathrm{~Pb}-211$ & $2.76 \mathrm{E}+01$ \\
\hline Co-60 & $3.61 \mathrm{E}-04$ & $\mathrm{~Pb}-212$ & $1.57 \mathrm{E}+00$ \\
\hline $\mathrm{Co}-60 \mathrm{~m}$ & $9.54 \mathrm{E}+01$ & $\mathrm{~Pb}-214$ & $3.71 \mathrm{E}+01$ \\
\hline Cs-135 & $8.25 \mathrm{E}-10$ & Pd-107 & $2.92 \mathrm{E}-10$ \\
\hline
\end{tabular}


Table A-10 Radionuclide Decay Constants (1/day) (Tuli 2005)

Radionuclide

Po-210

Po-211

Po-212

Po-213

Po-214

Po-215

Po-216

Po-218

Pt-193

Pu-236

Pu-237

$\mathrm{Pu}-238$

Pu-239

Pu-240

Pu-241

$\mathrm{Pu}-242$

$\mathrm{Pu}-243$

$\mathrm{Pu}-244$

Pu-246

$\mathrm{Ra}-222$

Ra-223

Ra-224

Ra-225

Ra-226

Ra-228

$\mathrm{Rb}-87$

Re-186

Re-186m

Re-187

Rn-218

$\mathrm{Rn}-219$

$\mathrm{Rn}-220$

Rn-222

Ru-97

Sb-126

Sb-126m

Sc-44

Se-79

Si-32

Sm-146

Sm-147

Sn-126

Sr-90

Ta-180
Decay Constant 4.98E-03

$1.16 \mathrm{E}+05$

$2.00 \mathrm{E}+11$

$1.64 \mathrm{E}+10$

$3.65 \mathrm{E}+08$

$3.36 \mathrm{E}+07$

$4.13 \mathrm{E}+05$

$3.22 \mathrm{E}+02$

$3.79 \mathrm{E}-05$

6.64E-04

$1.53 \mathrm{E}-02$

$2.16 \mathrm{E}-05$

7.87E-08

$2.89 \mathrm{E}-07$

$1.33 \mathrm{E}-04$

5.07E-09

$3.36 \mathrm{E}+00$

$2.38 \mathrm{E}-11$

$6.39 \mathrm{E}-02$

$1.58 \mathrm{E}+03$

6.06E-02

$1.91 \mathrm{E}-01$

4.64E-02

$1.19 \mathrm{E}-06$

3.31E-04

3.81E-14

$1.86 \mathrm{E}-01$

$9.49 \mathrm{E}-09$

4.61E-14

$1.71 \mathrm{E}+06$

$1.51 \mathrm{E}+04$

$1.08 \mathrm{E}+03$

$1.81 \mathrm{E}-01$

$2.48 \mathrm{E}-01$

$5.60 \mathrm{E}-02$

$5.20 \mathrm{E}+01$

$4.19 \mathrm{E}+00$

6.43E-09

$1.44 \mathrm{E}-05$

$1.84 \mathrm{E}-11$

$1.79 \mathrm{E}-14$

8.25E-09

6.57E-05

$1.58 \mathrm{E}-18$
Radionuclide

Ta-182

Tc-97

Tc-97m

Tc-98

Tc-99

Te-123

Th-226

Th-227

Th-228

Th-229

Th-230

Th-231

Th-232

Th-234

Ti-44

Tl-202

Tl-206

T1-207

Tl-208

Tl-209

U-230

U-232

U-233

U-234

U-235

U-236

U-237

U-238

U-240

V-49

Y-90

Zr-93
Decay Constant

6.04E-03

$4.50 \mathrm{E}-10$

$7.56 \mathrm{E}-03$

4.52E-10

8.99E-09

2.06E-20

$3.27 \mathrm{E}+01$

$3.71 \mathrm{E}-02$

9.94E-04

2.58E-07

$2.51 \mathrm{E}-08$

6.52E-01

$1.35 \mathrm{E}-13$

2.88E-02

$3.16 \mathrm{E}-05$

$5.65 \mathrm{E}-02$

$2.38 \mathrm{E}+02$

$2.09 \mathrm{E}+02$

$3.27 \mathrm{E}+02$

$4.61 \mathrm{E}+02$

3.32E-02

2.75E-05

$1.19 \mathrm{E}-08$

7.73E-09

2.70E-12

$8.10 \mathrm{E}-11$

1.03E-01

$4.25 \mathrm{E}-13$

$1.18 \mathrm{E}+00$

2.11E-03

2.59E-01

1.24E-09 
Table A-11 Specific Activities (Taylor 2006)

\begin{tabular}{|c|c|c|}
\hline Radionuclide & $\mathrm{Ci} / \mathrm{mole}$ & $\mathrm{Ci} / \mathrm{g}$ \\
\hline Ac-227 & $1.64 \mathrm{E}+04$ & 7.23E+01 \\
\hline Ag- $108 \mathrm{~m}$ & $8.16 \mathrm{E}+02$ & $7.56 \mathrm{E}+00$ \\
\hline Al-26 & $4.99 \mathrm{E}-01$ & $1.92 \mathrm{E}-02$ \\
\hline Am-237 & $2.57 \mathrm{E}+09$ & $1.09 \mathrm{E}+07$ \\
\hline Am-241 & $8.27 \mathrm{E}+02$ & $3.43 \mathrm{E}+00$ \\
\hline Am-243 & $4.85 \mathrm{E}+01$ & $2.00 \mathrm{E}-01$ \\
\hline $\mathrm{Be}-10$ & $2.37 \mathrm{E}-01$ & $2.37 \mathrm{E}-02$ \\
\hline Bi-210m & $1.18 \mathrm{E}-01$ & $5.60 \mathrm{E}-04$ \\
\hline Bk-247 & $2.59 \mathrm{E}+02$ & $1.05 \mathrm{E}+00$ \\
\hline Bk-249 & $3.95 \mathrm{E}+05$ & $1.59 \mathrm{E}+03$ \\
\hline C-14 & $6.24 \mathrm{E}+01$ & $4.46 \mathrm{E}+00$ \\
\hline $\mathrm{Ca}-41$ & $3.50 \mathrm{E}+00$ & $8.55 \mathrm{E}-02$ \\
\hline Cd-113 & 4.64E-11 & $4.10 \mathrm{E}-13$ \\
\hline Cf-249 & $1.02 \mathrm{E}+03$ & $4.08 \mathrm{E}+00$ \\
\hline Cf-250 & $2.73 E+04$ & $1.09 \mathrm{E}+02$ \\
\hline Cf-251 & $3.99 \mathrm{E}+02$ & $1.59 \mathrm{E}+00$ \\
\hline Cf- 252 & $1.35 \mathrm{E}+05$ & $5.35 \mathrm{E}+02$ \\
\hline $\mathrm{Cl}-36$ & $1.19 \mathrm{E}+00$ & 3.30E-02 \\
\hline $\mathrm{Cm}-241$ & $3.99 \mathrm{E}+06$ & $1.65 \mathrm{E}+04$ \\
\hline $\mathrm{Cm}-242$ & $8.00 \mathrm{E}+05$ & $3.30 \mathrm{E}+03$ \\
\hline $\mathrm{Cm}-244$ & $1.98 \mathrm{E}+04$ & $8.09 \mathrm{E}+01$ \\
\hline $\mathrm{Cm}-245$ & $4.21 \mathrm{E}+01$ & $1.72 \mathrm{E}-01$ \\
\hline $\mathrm{Cm}-246$ & $7.51 \mathrm{E}+01$ & $3.05 \mathrm{E}-01$ \\
\hline Cm-247 & $2.29 \mathrm{E}-02$ & $9.28 \mathrm{E}-05$ \\
\hline $\mathrm{Cm}-248$ & $1.02 \mathrm{E}+00$ & $4.13 \mathrm{E}-03$ \\
\hline $\mathrm{Cm}-250$ & $4.30 \mathrm{E}+01$ & $1.72 \mathrm{E}-01$ \\
\hline Сo-60 & $6.80 \mathrm{E}+04$ & $1.13 \mathrm{E}+03$ \\
\hline Cs-135 & $1.55 \mathrm{E}-01$ & $1.15 \mathrm{E}-03$ \\
\hline Es-253 & $6.37 \mathrm{E}+06$ & $2.52 \mathrm{E}+04$ \\
\hline $\mathrm{Fe}-60$ & $2.38 \mathrm{E}-01$ & $3.97 \mathrm{E}-03$ \\
\hline Gd- 152 & $3.31 \mathrm{E}-09$ & $2.18 \mathrm{E}-11$ \\
\hline $\mathrm{Ge}-68$ & $4.82 \mathrm{E}+05$ & $7.09 \mathrm{E}+03$ \\
\hline $\mathrm{H}-3$ & $2.90 \mathrm{E}+04$ & $9.67 \mathrm{E}+03$ \\
\hline Hf-182 & 4.01E-02 & $2.20 \mathrm{E}-04$ \\
\hline Hg-194 & $8.05 \mathrm{E}+02$ & $4.15 \mathrm{E}+00$ \\
\hline Ho-166m & $2.98 \mathrm{E}+02$ & $1.79 \mathrm{E}+00$ \\
\hline I-129 & $2.28 \mathrm{E}-02$ & $1.77 \mathrm{E}-04$ \\
\hline In-115 & $8.11 \mathrm{E}-10$ & $7.05 \mathrm{E}-12$ \\
\hline Ir-192m & $1.48 \mathrm{E}+03$ & $7.73 E+00$ \\
\hline K-40 & $2.86 \mathrm{E}-04$ & 7.15E-06 \\
\hline La-137 & $5.97 \mathrm{E}+00$ & $4.36 \mathrm{E}-02$ \\
\hline La-138 & $3.50 \mathrm{E}-06$ & $2.54 \mathrm{E}-08$ \\
\hline Lu-176 & $9.48 \mathrm{E}-06$ & $5.39 \mathrm{E}-08$ \\
\hline
\end{tabular}


Table A-11 Specific Activities (Taylor 2006)

\begin{tabular}{|c|c|c|}
\hline Radionuclide ${ }^{*}$ & $\mathrm{Ci} / \mathrm{mole}$ & $\mathrm{Ci} / \mathrm{g}$ \\
\hline $\mathrm{Mn}-53$ & $9.56 \mathrm{E}-02$ & $1.80 \mathrm{E}-03$ \\
\hline Mo-93 & $8.94 \mathrm{E}+01$ & $9.61 \mathrm{E}-01$ \\
\hline $\mathrm{Nb}-93 \mathrm{~m}$ & $2.22 \mathrm{E}+04$ & $2.39 \mathrm{E}+02$ \\
\hline $\mathrm{Nb}-94$ & $1.76 \mathrm{E}+01$ & $1.87 \mathrm{E}-01$ \\
\hline Ni-59 & $4.70 \mathrm{E}+00$ & $7.96 \mathrm{E}-02$ \\
\hline $\mathrm{Np}-236 \mathrm{a}$ & $2.32 \mathrm{E}+00$ & $9.84 \mathrm{E}-03$ \\
\hline $\mathrm{Np}-237$ & $1.66 \mathrm{E}-01$ & $7.02 \mathrm{E}-04$ \\
\hline $\mathrm{Pa}-230$ & $7.51 \mathrm{E}+06$ & $3.27 E+04$ \\
\hline $\mathrm{Pa}-231$ & $1.09 \mathrm{E}+01$ & $4.73 \mathrm{E}-02$ \\
\hline $\mathrm{Pb}-202$ & $6.80 \mathrm{E}+00$ & $3.36 \mathrm{E}-02$ \\
\hline $\mathrm{Pb}-205$ & $2.07 \mathrm{E}-02$ & $1.01 \mathrm{E}-04$ \\
\hline $\mathrm{Pb}-210$ & $1.61 \mathrm{E}+04$ & $7.67 \mathrm{E}+01$ \\
\hline Pd-107 & $5.50 \mathrm{E}-02$ & $5.14 \mathrm{E}-04$ \\
\hline Pt-193 & $7.14 \mathrm{E}+03$ & $3.70 \mathrm{E}+01$ \\
\hline $\mathrm{Pu}-237$ & $2.88 \mathrm{E}+06$ & $1.22 \mathrm{E}+04$ \\
\hline $\mathrm{Pu}-238$ & $4.07 \mathrm{E}+03$ & $1.71 \mathrm{E}+01$ \\
\hline $\mathrm{Pu}-239$ & $1.48 \mathrm{E}+01$ & $6.21 \mathrm{E}-02$ \\
\hline $\mathrm{Pu}-240$ & $5.45 \mathrm{E}+01$ & $2.27 \mathrm{E}-01$ \\
\hline $\mathrm{Pu}-241$ & $2.50 \mathrm{E}+04$ & $1.04 \mathrm{E}+02$ \\
\hline $\mathrm{Pu}-242$ & $9.56 \mathrm{E}-01$ & $3.95 \mathrm{E}-03$ \\
\hline $\mathrm{Pu}-244$ & 4.47E-03 & $1.83 \mathrm{E}-05$ \\
\hline $\mathrm{Ra}-226$ & $2.23 \mathrm{E}+02$ & $9.89 \mathrm{E}-01$ \\
\hline $\mathrm{Ra}-228$ & $6.23 \mathrm{E}+04$ & $2.73 E+02$ \\
\hline $\mathrm{Rb}-87$ & $7.18 \mathrm{E}-06$ & $8.25 \mathrm{E}-08$ \\
\hline Re-186m & $1.79 E+00$ & $9.61 \mathrm{E}-03$ \\
\hline Re-187 & 8.68E-06 & 4.64E-08 \\
\hline Ru-97 & $4.68 \mathrm{E}+07$ & $4.82 \mathrm{E}+05$ \\
\hline Se-79 & $1.21 \mathrm{E}+00$ & $1.53 \mathrm{E}-02$ \\
\hline $\mathrm{Si}-32$ & $2.71 E+03$ & $8.46 \mathrm{E}+01$ \\
\hline Sm-146 & $3.47 \mathrm{E}-03$ & $2.38 \mathrm{E}-05$ \\
\hline Sm-147 & 3.37E-06 & 2.29E-08 \\
\hline $\mathrm{Sn}-126$ & $1.55 \mathrm{E}+00$ & $1.23 \mathrm{E}-02$ \\
\hline Sr-90 & $1.24 \mathrm{E}+04$ & $1.37 \mathrm{E}+02$ \\
\hline Та-180 & $2.98 \mathrm{E}-10$ & $1.66 \mathrm{E}-12$ \\
\hline Tc-97 & $8.47 \mathrm{E}-02$ & $8.73 \mathrm{E}-04$ \\
\hline Tc-99 & $1.69 \mathrm{E}+00$ & $1.71 \mathrm{E}-02$ \\
\hline Te-123 & $3.89 \mathrm{E}-12$ & $3.16 \mathrm{E}-14$ \\
\hline Th-229 & $4.86 \mathrm{E}+01$ & $2.12 \mathrm{E}-01$ \\
\hline Th-230 & $4.74 \mathrm{E}+00$ & $2.06 \mathrm{E}-02$ \\
\hline Th-232 & $2.54 \mathrm{E}-05$ & $1.10 \mathrm{E}-07$ \\
\hline Ti-44 & $5.96 \mathrm{E}+03$ & $1.35 \mathrm{E}+02$ \\
\hline U-232 & $5.19 \mathrm{E}+03$ & $2.24 \mathrm{E}+01$ \\
\hline $\mathrm{U}-233$ & $2.25 \mathrm{E}+00$ & $9.64 \mathrm{E}-03$ \\
\hline U-234 & $1.46 \mathrm{E}+00$ & $6.22 \mathrm{E}-03$ \\
\hline
\end{tabular}


Rev. 0

\section{Table A-11 Specific Activities (Taylor 2006)}

\begin{tabular}{lrr}
$\quad$ Radionuclide & \multicolumn{1}{c}{ Ci/mole } & Ci/g \\
U-235 & $5.08 \mathrm{E}-04$ & $2.16 \mathrm{E}-06$ \\
U-236 & $1.53 \mathrm{E}-02$ & $6.47 \mathrm{E}-05$ \\
U-238 & $8.00 \mathrm{E}-05$ & $3.36 \mathrm{E}-07$ \\
V-49 & $3.97 \mathrm{E}+05$ & $8.10 \mathrm{E}+03$ \\
Zr-93 & $2.34 \mathrm{E}-01$ & $2.51 \mathrm{E}-03$
\end{tabular}

* Parents and daughters in groundwater transport calculations with half-lives greater than 5 years. 
WSRC-STI-2006-00162

Rev. 0 
WSRC-STI-2006-00162

Rev. 0 


\section{Distribution:}

\section{Savannah River Site}

$\begin{array}{ll}\text { M. J. Ades } & 705-3 \mathrm{C}, \mathrm{Rm} .122 \\ \text { S. E. Aleman } & 773-42 \mathrm{~A}, \mathrm{Rm} .147 \\ \text { B. T. Butcher } & 773-43 \mathrm{~A}, \mathrm{Rm} .216 \\ \text { L. B. Collard } & 773-43 \mathrm{~A}, \mathrm{Rm} .207 \\ \text { J. R. Cook } & 773-43 \mathrm{~A}, \mathrm{Rm} .209 \\ \text { K. P. Crapse } & 773-43 \mathrm{~A}, \mathrm{Rm} .212 \\ \text { M. E. Denham } & 773-42 \mathrm{~A}, \mathrm{Rm} .218 \\ \text { G. P. Flach } & 773-42 \mathrm{~A}, \mathrm{Rm} .211 \\ \text { W. T. Goldston } & 705-3 \mathrm{C}, \mathrm{Rm} .105 \\ \text { J. C. Griffin } & 773 \mathrm{~A}, \mathrm{Rm} . \mathrm{A}-231 \\ \text { L. L. Hamm } & 773-42 \mathrm{~A}, \mathrm{Rm} .145 \\ \text { T. Hang } & 773-42 \mathrm{~A}, \mathrm{Rm} .152 \\ \text { R. A. Hiergesell } & 773-42 \mathrm{~A}, \mathrm{Rm} .251 \\ \text { D. I. Kaplan } & 773-43 \mathrm{~A}, \mathrm{Rm} .215 \\ \text { M. A. Phifer } & 773-42 \mathrm{~A}, \mathrm{Rm} .252 \\ \text { D. F. Sink, Jr. } & 704-56 \mathrm{E}, \mathrm{Rm} .1 \\ \text { W. E. Stevens } & 773-\mathrm{A}, \mathrm{Rm} . \mathrm{A}-261 \\ \text { R. F. Swingle } & 773-42 \mathrm{~A}, \mathrm{Rm} .122 \\ \text { G. A. Talyor } & 773-41 \mathrm{~A}, \mathrm{Rm} .156 \\ \text { E. L. Wilhite } & 773-43 \mathrm{~A}, \mathrm{Rm} .214 \\ \text { EA\&PM File (2 copies) } & 773-43 \mathrm{~A}, \mathrm{Rm} .213 \\ \text { STI (3 copies) } & 703-43 \mathrm{~A}\end{array}$

